\title{
NUMERICAL MODELLING OF GROUND PENETRATING RADAR FOR POTASH MINE SAFETY
}

\author{
A Thesis \\ Submitted to the Faculty of Graduate Studies and Research \\ In Partial Fulfillment of the Requirements \\ For the Degree of
}

Master of Applied Science

in

Electronic Systems Engineering

University of Regina

By

Victor Chigozie Okonkwo

Regina, Saskatchewan

June, 2019

(C) Copyright 2019: V.C. Okonkwo 


\section{UNIVERSITY OF REGINA \\ FACULTY OF GRADUATE STUDIES AND RESEARCH SUPERVISORY AND EXAMINING COMMITTEE}

Victor Chigozie Okonkwo, candidate for the degree of Master of Applied Science in Electronic Systems Engineering, has presented a thesis titled, Numerical Modelling of Ground Penetrating Radar for Potash Mine Safety, in an oral examination held on June 17,2019 . The following committee members have found the thesis acceptable in form and content, and that the candidate demonstrated satisfactory knowledge of the subject material.

External Examiner: $\quad$ *Dr. Anh Dinh, University of Saskatchewan

Supervisor: $\quad$ Dr. Raman Paranjape, Electronic Systems Engineering

Committee Member: Dr. Paul Laforge, Electronic Systems Engineering

Committee Member: Dr. Lei Zhang, Electronic Systems Engineering

Committee Member: Dr. Hairuo Qing, Department of Geology

Chair of Defense: $\quad$ Dr. Kin Choong Yow, Software Systems Engineering

*via Video-Conference 


\section{Abstract}

This thesis presents a software tool which simulates the geological stratigraphy of a potash mine which is then used with gprMax (public domain Ground Penetrating Radar (GPR) simulation software) to examine and evaluate the effectiveness of auto-picking algorithms. The system is used to simulate the GPR response from clay seams in the roof of potash mining rooms. As it is extremely onerous to obtain in-situ data that captures all possible normal and anomalous geological conditions present in the mine roof, earth models are generated which accurately represents the geology of the mine. In particular, random clays in the mine roof can negatively affect the performance of auto-picking algorithms. These earth model simulations can be used to present these random clays accurately

gprMax is an open source software that simulates Electro-Magnetic (EM) wave propagation in materials in order to support better understanding of the use of GPR in various application domains. Currently, GPR machines are being used in the potash mine as a secondary inspection of salt (roof) thickness. The goal of this thesis is to validate the ability to use gprMax with an effective earth model to generate realistic GPR signals to test/evaluate auto-picking algorithms. The use of simulated data in comparison to the experimental (actual) data and generate test bed models for an auto-picking algorithm has many benefits. The synthetic data is generated by gprMax using the Finite Difference Time Domain (FDTD) methodology. An effective methodology to develop and test robust auto- 
picking algorithms is created using simulated GPR signals because the ground truth is known from the earth models.

Additionally, in this thesis results from both an industry standard auto-picking algorithm and a new auto-picking algorithm, called Clustered Ratio Derivative (CRD), are presented for the mine roof application. Finally, in this thesis we take advantage of cloud computing resources in order to execute this work and our use if this technology is summarized.

Keywords: gprMax, Ground Penetrating Radar, Electro-Magnetic wave, Finite Difference Time Domain, auto-picking algorithm, simulation. 


\section{Acknowledgements}

I want to say a big thank you to my supervisor Prof. Raman Paranjape for accepting me as his research student. He gave me a platform to develop myself and become a better person. Through him I have grown in knowledge. He has equally been a friend too and his advices has helped me in life. Thank you Raman for the financial support, guidance and encouragement, I am grateful.

My thesis wouldn't have been successful without the financial support from Nutrien Ltd and Mitacs. Special thanks to Matthew van der Berghe who I have reported closely on the progress of my research.

I want to thank the rest of my committee members, Dr. Paul Laforge, Dr. Lei Zhang, Dr. Hairuo Qing and my external examiner Dr. Anh Dinh for accepting to review my thesis and giving their insightful comments.

I also would like to extend my appreciation to my friends, most especially Tokini, Mildred and Ifunanya for going far and beyond in helping to review my thesis.

Lastly, I want to specially thank and dedicate my thesis to my mum, dad and sister for their prayers, encouragements and belief in me. I couldn't have achieved this without any of you. God bless you all. 


\section{Table of Contents}

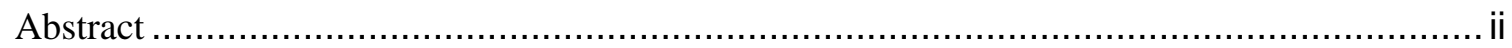

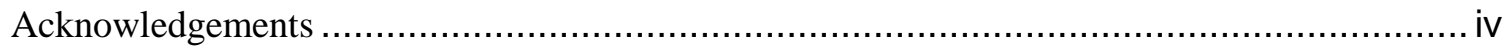

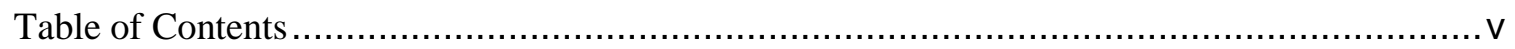

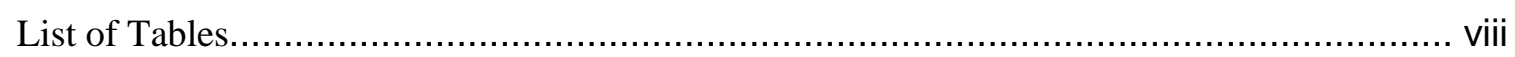

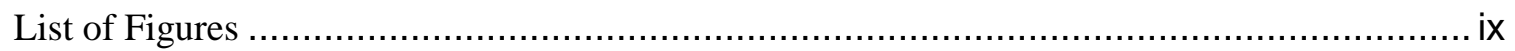

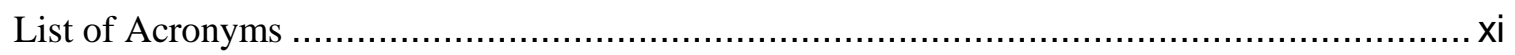

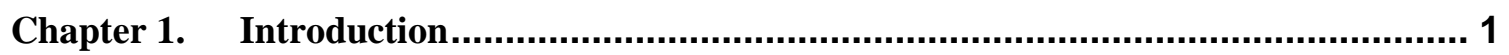

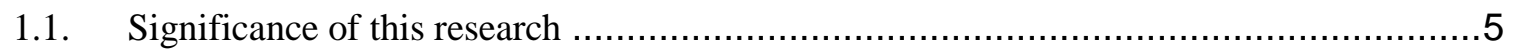

1.2. Thesis outline

Chapter 2. GPR and Auto-Picking Techniques ............................................................. 7

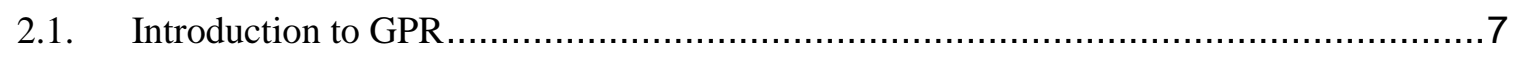

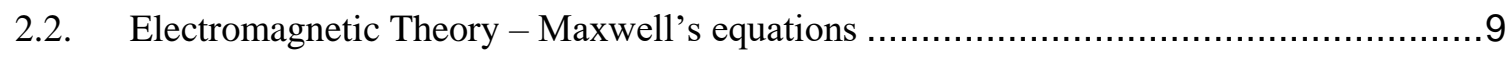

2.3. Numerical modeling using FDTD method …………....................................11

2.4. EM wave propagation with subsurface materials ............................................14

2.4.1. Wave Propagation - Reflection, Refraction, Diffraction.....................................15

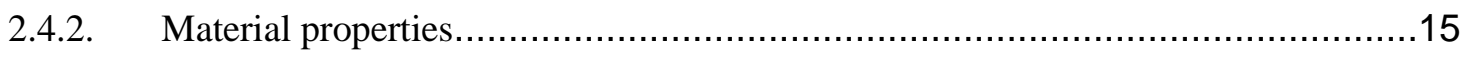

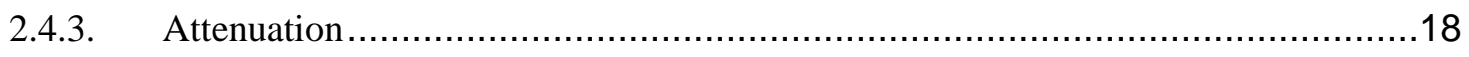

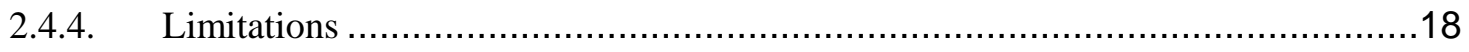

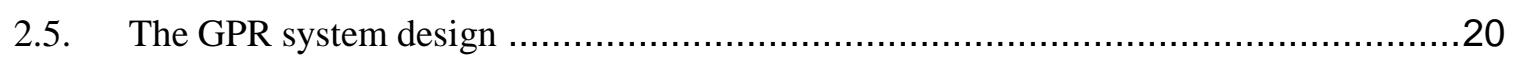

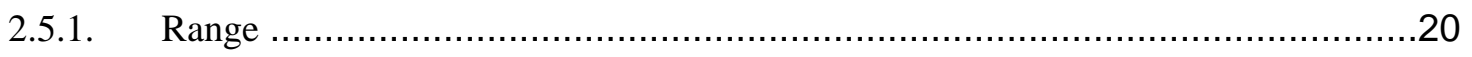

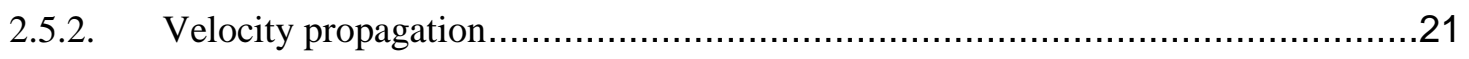




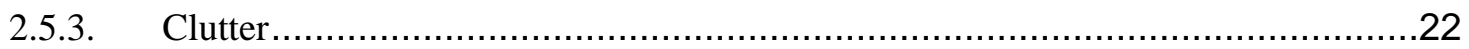

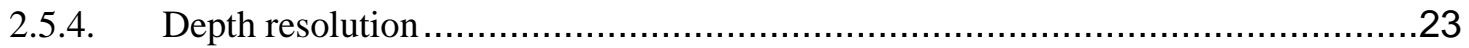

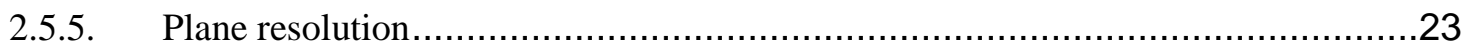

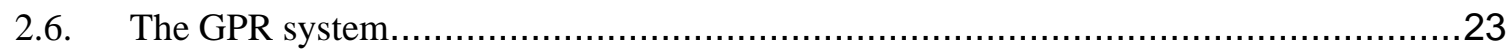

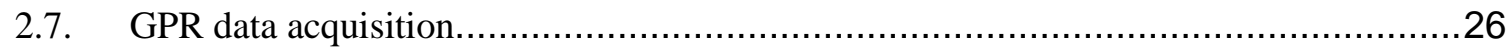

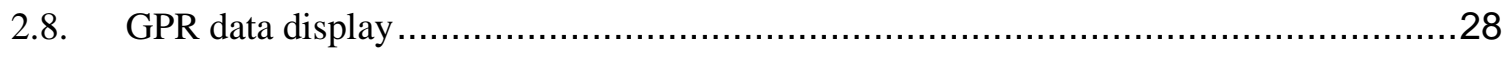

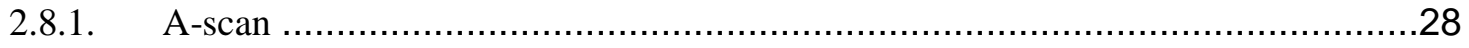

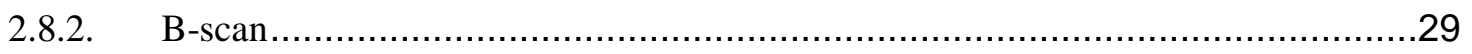

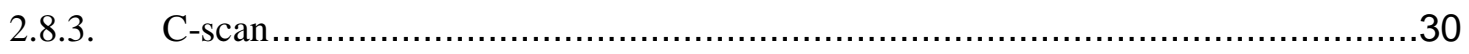

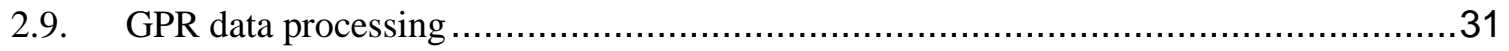

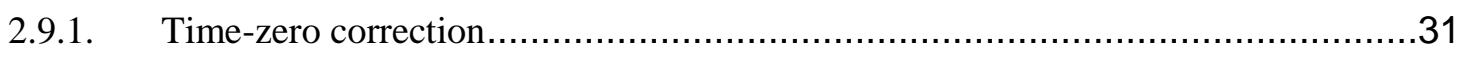

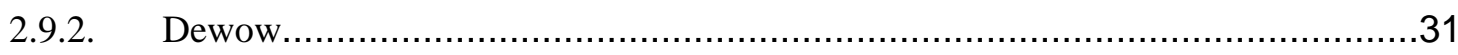

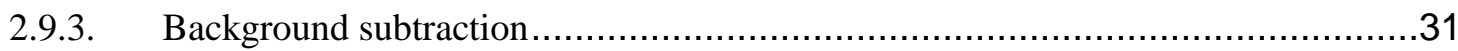

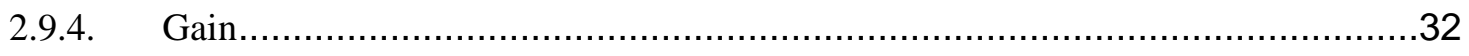

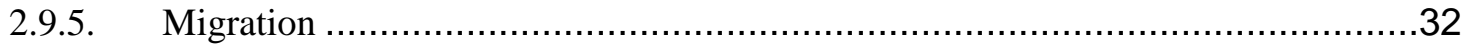

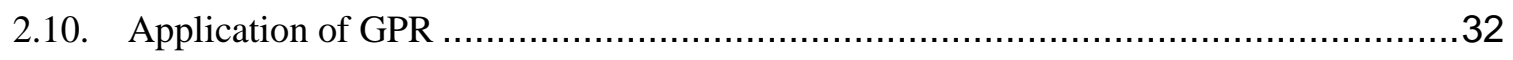

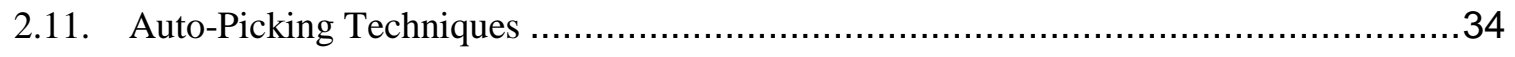

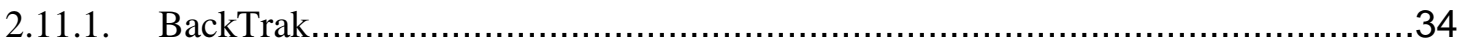

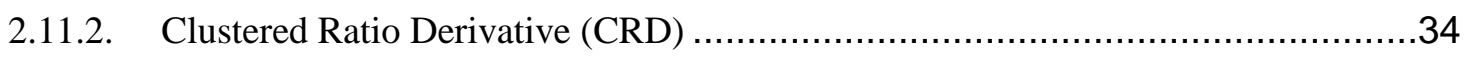

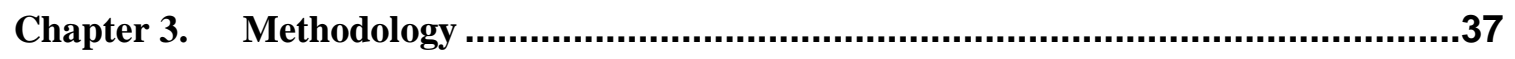

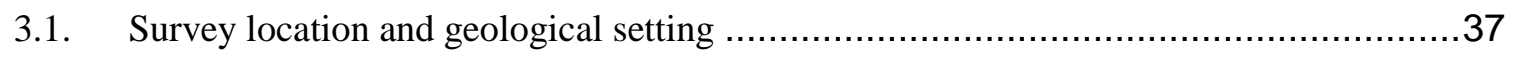

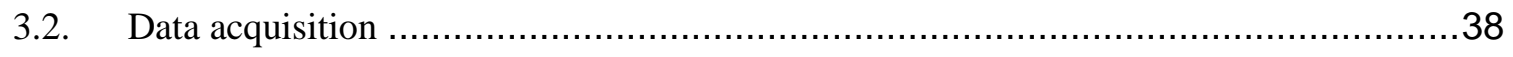

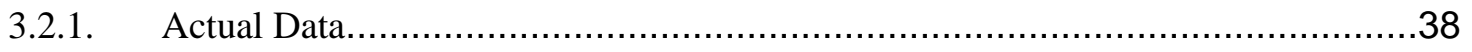




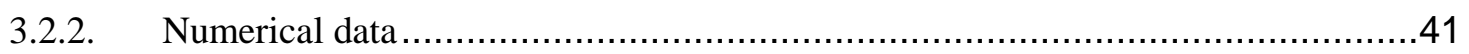

3.3. Random generation of 414 clay seam profile.............................................. 46

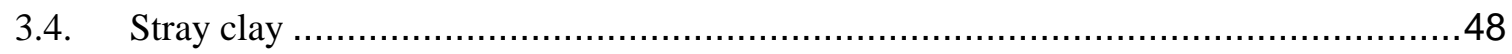

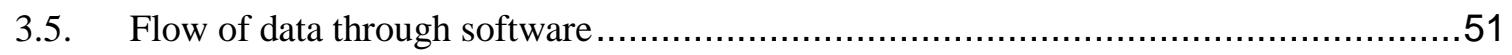

Chapter 4. Results and Analysis............................................................................54

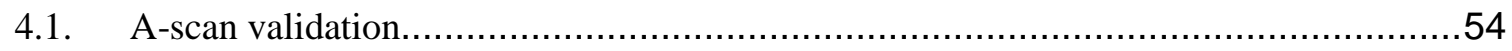

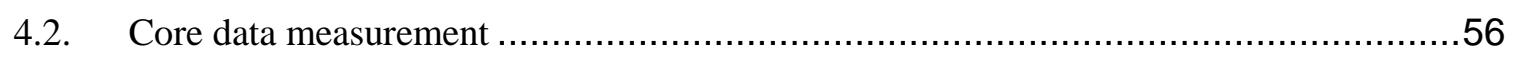

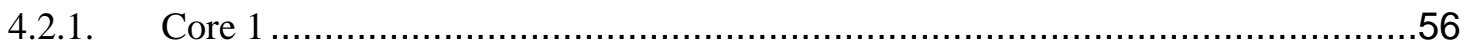

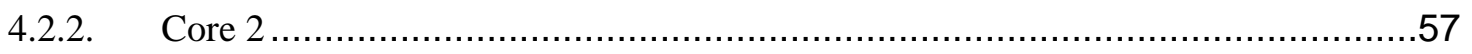

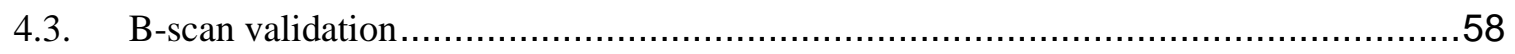

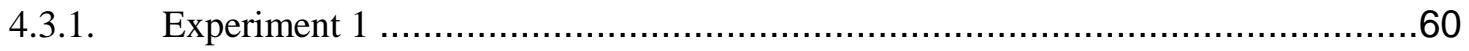

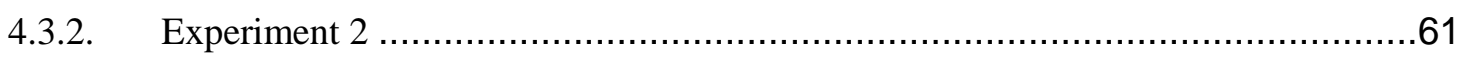

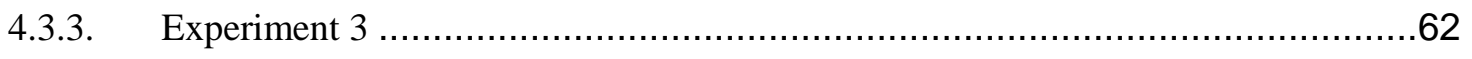

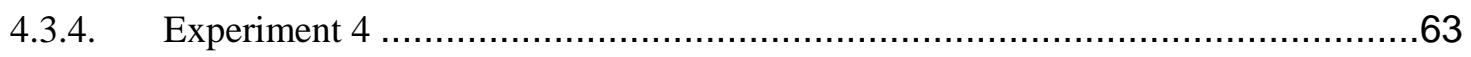

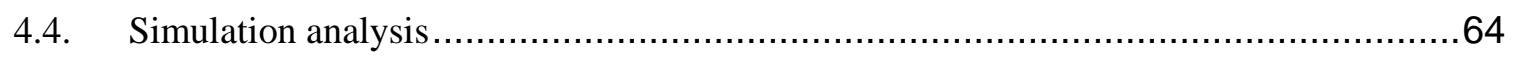

Chapter 5. Conclusion and Future Work...........................................................67

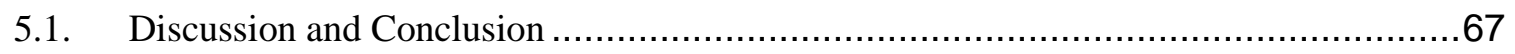

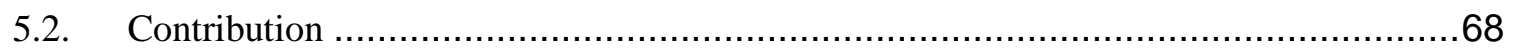

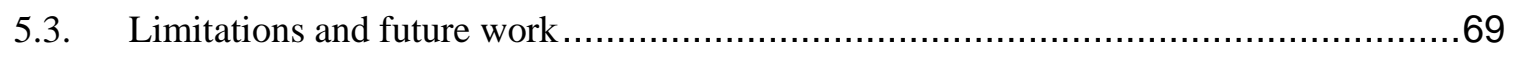

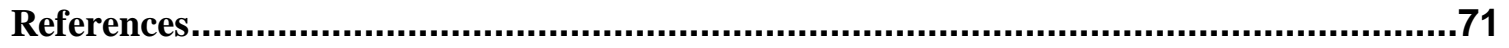

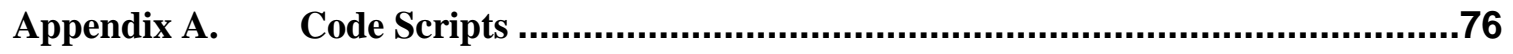




\section{List of Tables}

Table 2.1. Electrical properties of salt and related targets found in potash mine [31].........16

Table 2.2. Typical Applications of Ground Penetrating Radar [10] ....................................33

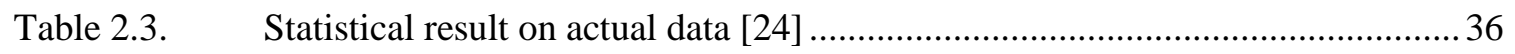

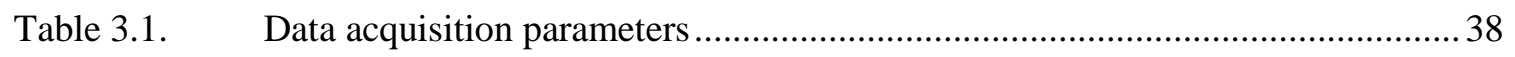

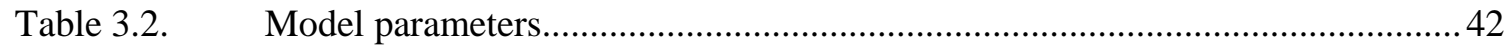

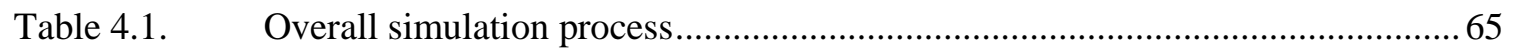

Table 4.2. Simulation time of an ordinary PC without GPU vs. a virtual PC with 1-GPU for

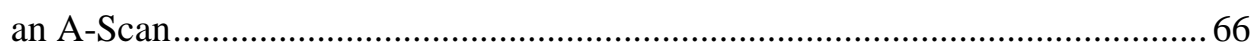




\section{List of Figures}

Fig. 1.1. An illustration of a potash mining machine in Upper Patience Lake Member (A zone) of the Prairie Evaporite Formation. [29] .................................................. 5

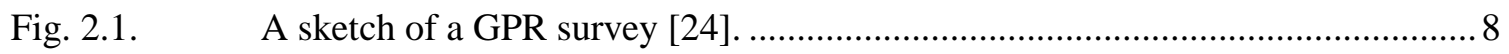

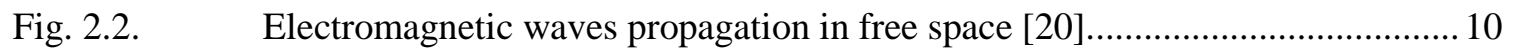

Fig. 2.3. FDTD Yee Cell indicating electric and magnetic field directions in 3D [21] ... 12

Fig. 2.4. Velocity propagation against relative dielectric permittivity [8]......................22

Fig. 2.5. Interaction between the transmitter and receiver of an antenna.........................24

Fig. 2.6. Sensors \& Software $1000 \mathrm{MHz}$ PulseEkko GPR [23] ....................................2

Fig. 2.7. A schematic representation of a common offset method.................................27

Fig. 2.8. A schematic representation of a common midpoint (CMP) survey method.......27

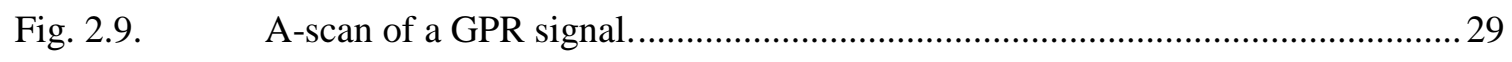

Fig. 2.10. Generation of a B-scan. The wiggle plot on the right shows the combination of series of A-scan with the profile of the target..................................................2 29

Fig. 2.11. Combination of several B-scan to form a C-scan........................................... 30

Fig. 2.12. A flowchart of the CRD algorithm process on GPR Data. .............................. 35

Fig. 3.1. Kubota ATV used for GPR survey.......................................................... 39

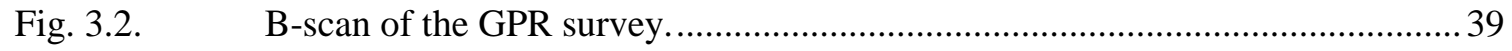

Fig. 3.3. Nutrien Geophysicist's interpretation of 414 Clay seam from GPR data collected using a Kubota (GPR-RTV) ................................................................ 40

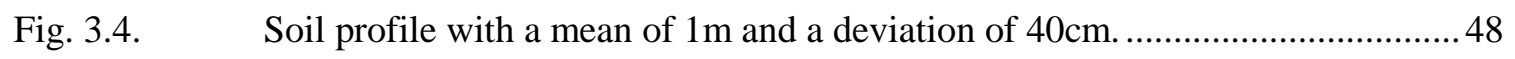


Fig. 3.5. Example of gprMax model with stray-clay 1 visualized with Paraview.

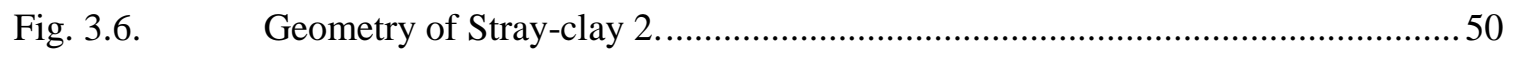

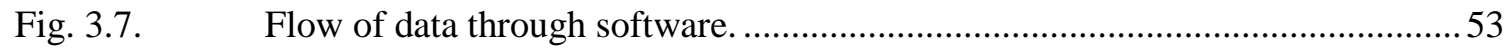

Fig. 4.1. (a) gprMax model. (b) A-Scan comparison between the actual and synthetic data of salt back thickness. It can be observed that the synthetic signal return is very

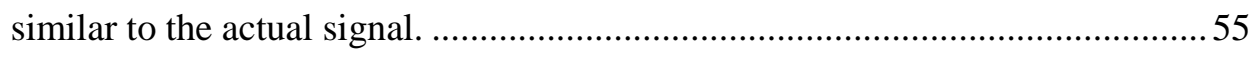

Fig. 4.2. Core-1 data with the corresponding A-scan................................................... 57

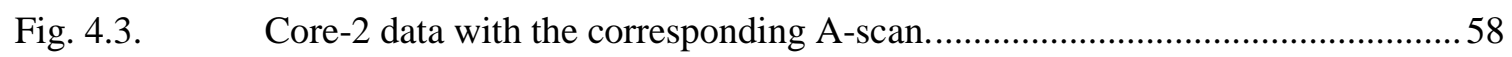

Fig. 4.4. Results of auto-picking techniques applied to the actual data. (a) BackTrak picks imported into Matlab. (b) CRD algorithm plotted in Matlab.............................59

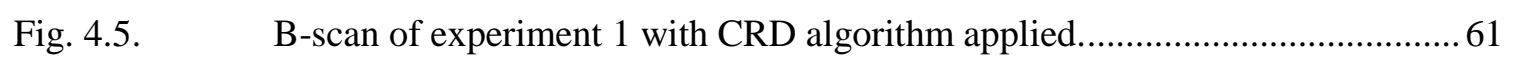

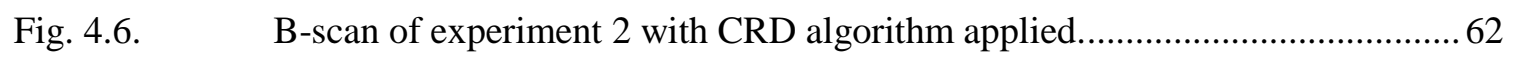

Fig. 4.7. B-scan of experiment 3 with CRD algorithm applied...................................63

Fig. 4.8. B-scan of experiment 4 with CRD algorithm applied......................................... 64 


\section{List of Acronyms}

1D

2D

3D

ABC

AGC

Auto-Picking

CFL

CMP

$\mathrm{CO}$

CRD

DBSCAN

DTA

EM

FDTD

GPR

PDE

PSD

SCR

SD

SEC

SNR

STA

TE

TM

TWTT
One Dimensional

Two Dimensional

Three Dimensional

Absorbing Boundary Condition

Automatic Gain Control

Automatic Picking

Courant, Freidrichs and Lewy

Common Mid-Point

Common Offset

Clustered Ratio Derivative

Density-based Spatial Clustering of Application with Noise

Delayed Term Average

Electro-Magnetic

Finite Difference Time Domain

Ground Penetrating Radar

Partial Differential Equation

Power Spectrum Density

Signal to Clutter Ratio

Standard Deviation

Spreading and Exponential Compensation

Signal to Noise Ratio

Short Term Average

Transverse Electric

Transverse Magnetic

Two Way Travel Time 


\section{Chapter 1.}

\section{Introduction}

Potash is a mineral used in producing fertilizers. These fertilizers are constantly produced to meet the needs of farmers in Saskatchewan. Therefore, the need for continuous mining of potash is necessary. The mining of potash possess some safety concerns, which leads to the study of numerical modelling for mine safety application. However, it important to understand the geological formation of potash in Saskatchewan. About four hundred (400) million years ago, deposits of potash formed underground by the evaporation of an ancient inland sea [1]. The topography behind the formation of potash in Saskatchewan involves three rock formation. These are: the Dawson Bay Formation, the Prairie Evaporite Formation, and the Winnipegosis formation [1]. The potash bed is located in the Prairie Evaporite Formation in between the Dawson Bay Formation and Winnipegosis Formation [27] [28]. In Saskatchewan, the conventional mining of potash occurs within a $500 \mathrm{~km}$ distance between Saskatoon region and the borders of Manitoba [2]. There are currently eight mines using the conventional mining technique. Five out of the eight mines are located within 100km radius of Saskatoon and three of them (Allan, Cory and Lanigan) are owned and operated by Nutrien Limited. Potash is continuously bored from the Upper Patience Lake Potash member [1] (at the Prairie Evaporite formation) of these mines. The mining process is automated with an attached conveyor belt to convey the bored potash [3]. During the boring process, the 
'Stress relief' mining method is applied. This method allows the outer mining rooms of the stress relief pattern to fail and collapse. The collapse of these rooms helps to shed the stress to neighboring rooms or openings in the mine [4] [5]. These stress reduction is useful in mining regions like Saskatchewan where the clay seam are less stable.

Mine safety has continually been a priority during mining operations. The instability of the mining roof during and after mining is a recurring problem in the mine due to limiting factors such as soft rock like potash that creeps when subjected to a high amount of stress [6]. Also thinning clay seam may give rise to bifurcation and generation of unstable salt-back (salt-back is referred to as the deposit of halite or salt between the mined roof and the 414 clay seam (Fig. 1.1)). Engineers have carefully designed a stress relief mining method that has been adopted during the boring process to create roof support in form of pillars (i.e. Pillars of potash that are never to be mined). In general, during mining of potash, the first or primary method of inspection by the mine operator is to observe the geological stratigraphy on the wall of the mining room visible to them. These mine operators have the ability to perceive potential or peculiar ground conditions that are considered unsafe. They rely on noticeable geological markers located above the mining room (i.e. wash-out, air-gap in roof) that become obvious in the event of an irregular ground condition. Some of these anomaly indicators show the presence of a compressed clay seam, salt intrusions, or distributed geology. In reference to an incidence (fall-ofground) that occurred in February 2013 at the Cory mine [2], the mining room had no visible stratigraphic marker indicating an anomaly of a thinning salt back in the mining room. Therefore a rock-bolt could not be installed because the geology present was considered normal. 
In order to prevent further occurrences, an upward-facing Ground Penetrating Radar (GPR) device was mounted on the mining machines as a secondary inspection method [2] [32]. The GPR is a non-destructive technique that emits electromagnetic energy in form of radio waves into the ground/roof of a potash mine for roof safety assessment. These upward-facing GPR systems monitor the salt-back, the distance from roof to 414 Clay Seam, which is normally $1 \mathrm{~m}$ thick. Fig. 1.1 shows a pictorial representation of the associated potash mining stratigraphy and also labels various clay seams in the geology including the 414 clay seam which is the marker that the boring machine must cut below in Saskatchewan mines. Normally it is expected that boring activity will always be at least $0.5 \mathrm{~m}$ below the 414 clay seam such that a sufficiently thick layer of salt will form the mining room roof so that the structural integrity of the mining room remains intact. In addition, the Fig. 1.1 presents a graphic of stray clays, in which the stray clays gives false reflection of the actual 414 clay seam which makes it difficult for the auto-picking algorithm (auto-picking is referred to as automatic picking algorithm of the 414 clay seam without human intervention) to keep track of. The operator of the mining machine sees the real-time display of the GPR returned signal and the output of an auto-picking algorithm which estimates the position of the clay seam above the mining room roof. Using this information, the operator makes operational safety decisions either to proceed with mining activity, provide additional roof support, or even stop mining.

There is a desire to improve the existing auto-picking algorithm which leads to the study of GPR simulators. Actual GPR data can be difficult to obtain because the GPR data has to be collected first and then, through a process of cutting rock core samples, the actual geology can be confirmed. It is generally not possible to obtain consistent samples of this 
type of data with varying characteristics of known ground truth (actual depth of 414 clay seam from the roof) from the actual geological structures (i.e. GPR and rock-core data of these 'stray clays'). However, the use of numerical modeling - a digital way of representing real world problems or scenarios using a computer - solves this problem.

The purpose of this thesis is to validate the ability to generate synthetic GPR signals that are similar to actual mine data to further develop series of test bed data using a GPR simulator like gprMax and an earth modeling system that emulates typical mining room conditions for continuous improvement of the auto-picking algorithm. gprMax [35], an open source GPR simulator software is utilized in this research to create various geological earth models to develop increasingly realistic, complex and relevant GPR data. These models enable us to test the effectiveness of auto-picking algorithms designed for tracking the 414 clay seam during mining to ensure mine room safety. gprMax is based on the methods of finite-difference time-domain (FDTD) [35] approximations to develop a numerical solver for the well-known Maxwell's equations. Computation, with the associated time required, is an essential part of the general modeling process. This research therefore uses an industry leading technique of cloud computing for the modelling and simulation processes. 


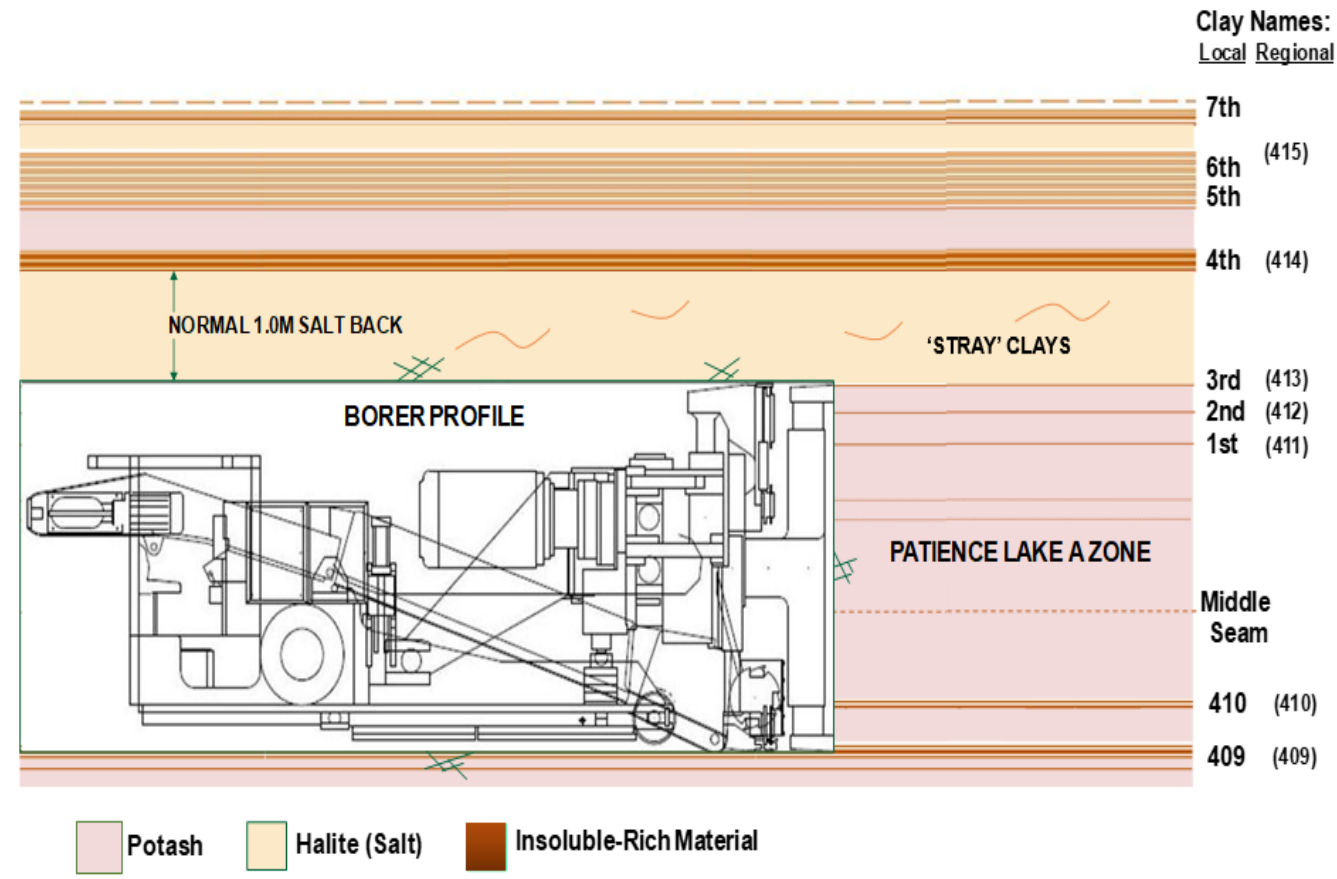

Fig. 1.1. An illustration of a potash mining machine in Upper Patience Lake Member (A zone) of the Prairie Evaporite Formation. [29].

\subsection{Significance of this research}

1. To develop a numerical software tool for simulating the geological stratigraphy of a potash mining room with 'stray clays' within the salt back.

2. Using the developed model to generate GPR signals that represents the same signals of the core data.

3. Using the software tool to generate realistic earth models of the geophysicist's interpretation of the clay seam and verifying them with the actual GPR data.

4. Generate test bed data of varying volume of 'stray clay' to evaluate the performance of auto-picking algorithms. 


\subsection{Thesis outline}

- Chapter 1 introduces the overall purpose and significance of this thesis.

- Chapter 2 reviews the theory behind GPR and the numerical modeling framework using the FDTD method. It also describes the data acquisition and interpretation processes, including the review of auto-picking techniques applicable to potash mine.

- Chapter 3 describes the methods employed in generating test bed synthetic data sets for auto-picking algorithm.

- Chapter 4 presents the analysis and validation of synthetic data with the rock-core measurements and geophysicist's interpretation of the actual data.

- Chapter 5 summaries the discussion and conclusion drawn from this thesis. A future work is also proposed. 


\section{Chapter 2.}

\section{GPR and Auto-Picking Techniques}

This chapter gives an overview of the physics behind Ground Penetrating Radar (GPR) signal generation, numerical modeling of GPR signals using the finite difference time domain (FDTD) method and the factors that affects the generation of GPR signals with adequate signal to noise ratio (SNR). It also describes how GPR data is acquired, the processing techniques employed, and the various forms of GPR data display. This chapter also introduces auto-picking algorithms applied to potash mine. These algorithms are used in validating the simulated (synthetic) data.

\subsection{Introduction to GPR}

GPR application is a quick developing field that has huge advancement in the improvement of theories, techniques and innovation in the course of the last $15-20$ years [9]. GPR has been used in various applications including: archeology, glaciology, mining, military, civil engineering and other industries [10].

GPR is a non-destructive technique that consist of a transmitting antenna that induces electromagnetic (EM) energy in form of radio waves - (with a frequency within 10 to $1000 \mathrm{MHz}$ range) [11] - into the ground for target detection. GPR works on the principle of scattering EM waves which is reflected back by any change in characteristics 
of the target. The EM wave is partly reflected back to the receiver of the antenna upon encountering a target as it goes from one medium to another. The amplitude of the reflected signal is dependent upon the difference in dielectric constant between the two mediums. One of the limiting factors of the EM wave is the signal attenuation, as higher frequencies provides less depth information with higher resolution and lower frequencies provides more depth information with lower resolution.

Fig. 2.1 shows a general GPR survey sketch of how GPR data is generated. The result of the survey displays the earth profile, with the point reflector inform of a hyperbola. The mounted antenna transmits and receives the EM wave at discrete time steps, moving from left to right it collects series of impulse radar together to produce a radargram for the operator carrying out a ground survey. Based upon the GPR data, the operator makes further decisions (to continue or stop boring, in the case of potash mining).

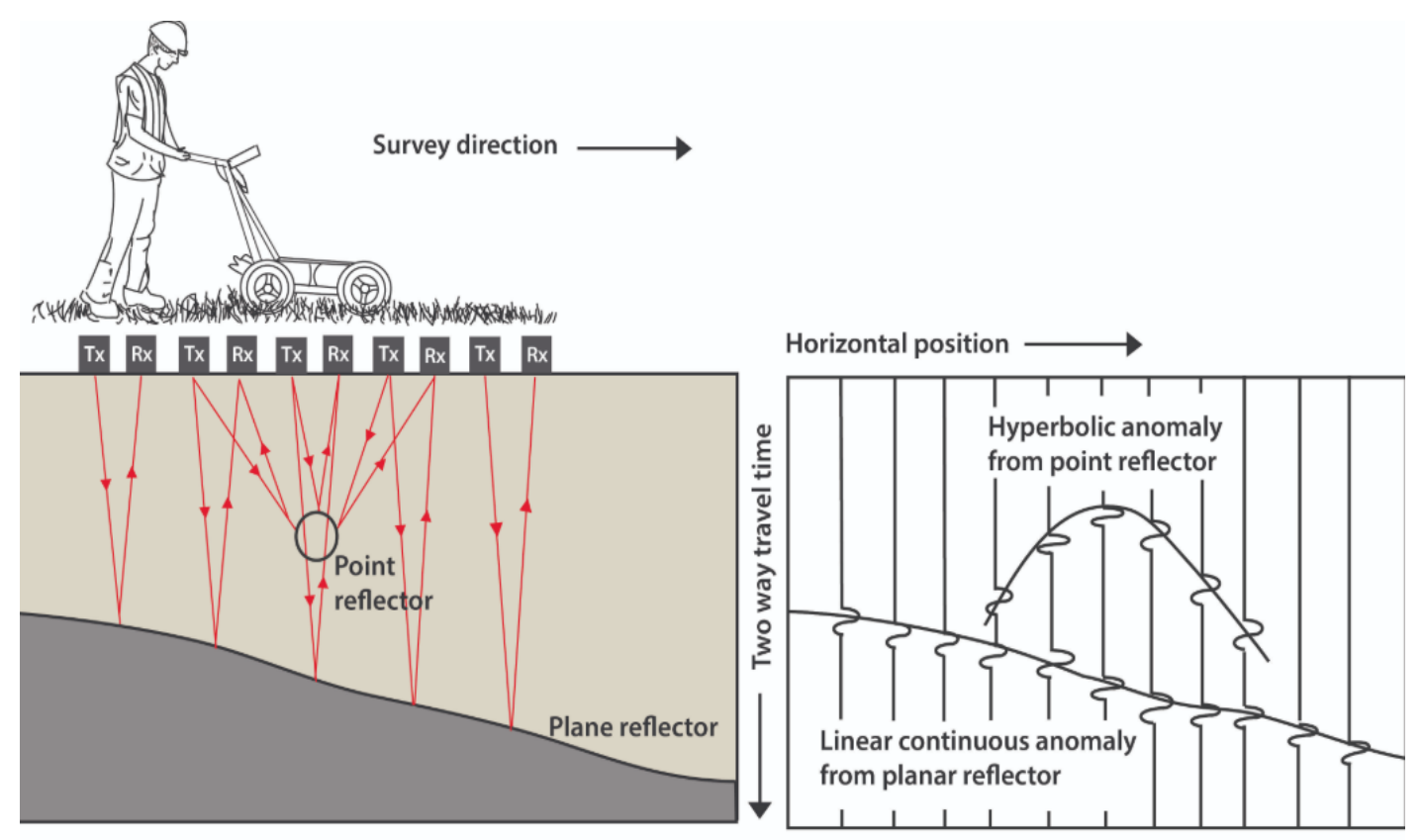

Fig. 2.1. A sketch of a GPR survey [24]. 
The work by Daniels [8] shows that successful GPR operations rely on the following conditions:

a. Effective coupling of GPR device with the ground.

b. Satisfactory penetration of EM waves through the ground with less attenuation with respect to the target depth.

c. Adequate amount of back reflected EM waves upon target detection.

d. Low noise level in the detected signal received.

It is important to note that integration of GPR data with other mine roof safety assessment methods helps to reduce uncertainty during potash mining.

\subsection{Electromagnetic Theory - Maxwell's equations}

Electromagnetic theory serves as the foundation for generating GPR signals. Seismic data is similar to GPR data but are implemented with acoustic energy while GPR data makes use of electromagnetic energy. GPR represents a subset of the electromagnetic field. The EM wave is governed by the well-known Maxwell's equations which mathematically describes the relationship between the electromagnetic physics and the material properties of the medium.

The governing mathematical equations for the electromagnetic field expressed in equations $2.1-2.4$ are derived as a general form for Faraday's law, Ampere's law, Gauss law for electric and magnetic fields respectively [19] [35] : 


$$
\begin{aligned}
& \bar{\nabla} \times \overline{\mathrm{E}}=-\frac{\partial \bar{B}}{\partial t} \\
& \bar{\nabla} \times \overline{\mathrm{H}}=\frac{\partial \bar{D}}{\partial t} \\
& \bar{\nabla} \cdot \overline{\mathrm{D}}=q \\
& \bar{\nabla} \cdot \overline{\mathrm{B}}=0
\end{aligned}
$$

Where:

$\overline{\mathrm{E}}-$ Electric field strength vector

$\overline{\mathrm{B}}-$ Magnetic flux density vector

$\overline{\mathrm{D}}$ - Electric displacement vector

$\overline{\mathrm{H}}-$ Magnetic field intensity vector

$q$ - Electric charge density

The above Maxwell's equations describe the relation between the electric and the magnetic field, which induces each other and are always perpendicular to each other (Fig. 2.2).

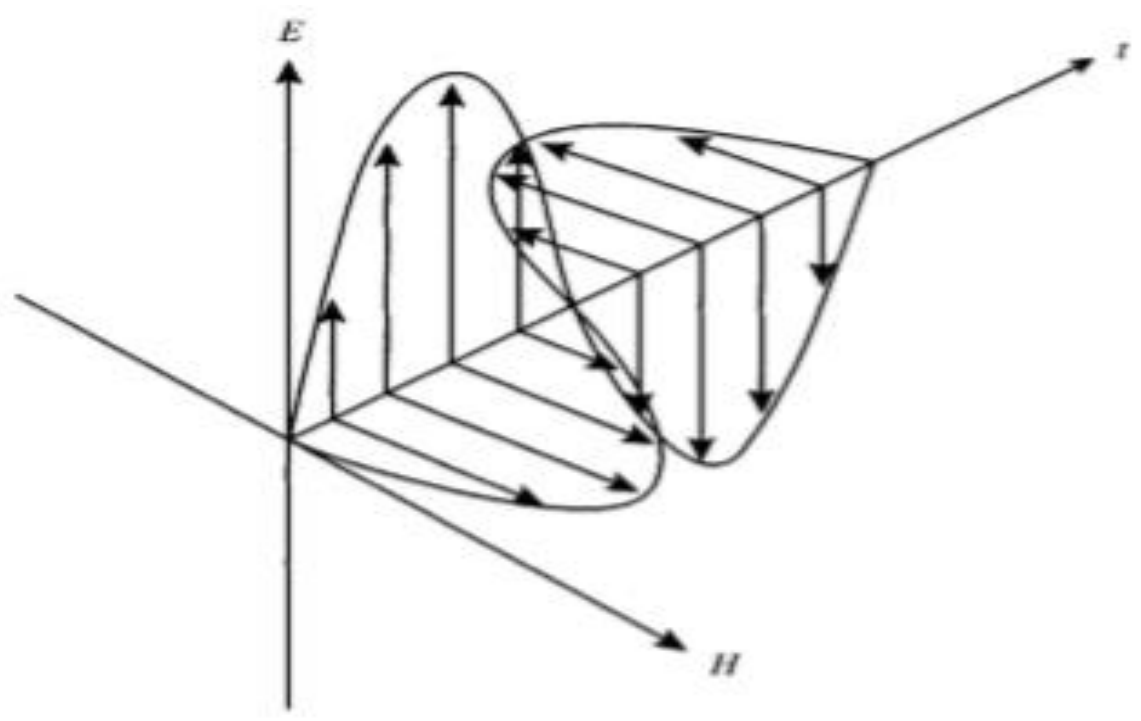

Fig. 2.2. Electromagnetic waves propagation in free space [20]. 
The definition of the electric current density to account for the electric loss mechanisms and an equivalent magnetic current density for the magnetic loss mechanisms and their substitution into the curl equations yields [19] [35]:

$$
\begin{aligned}
& \overline{J_{e}}=\sigma \overline{\mathrm{E}} \\
& \overline{J_{m}}=\rho^{\prime} \overline{\mathrm{H}} \\
& \frac{\partial \bar{H}}{\partial \mathrm{t}}=-\frac{1}{\mu} \nabla \times \overline{\mathrm{E}}-\frac{\rho^{\prime}}{\mu} \overline{\mathrm{H}} \\
& \frac{\partial \overline{\mathrm{E}}}{\partial \mathrm{t}}=-\frac{1}{\epsilon} \nabla \times \overline{\mathrm{H}}-\frac{\sigma}{\epsilon} \overline{\mathrm{E}}
\end{aligned}
$$

Where $\overline{J_{e}}-$ Electric current density vector, $\bar{J}_{m}-$ Magnetic current density vector, $\epsilon$ is the dielectric permittivity, $\sigma$ the electrical conductivity, $\mu$ the magnetic permeability and $\rho^{\prime}$ is the magnetic resistivity [22].

\subsection{Numerical modeling using FDTD method}

In order to understand and improve potash mine roof assessment using GPR, roof coring (traditional borings of mine roof) is necessary. However, it is onerous and difficult to consistently collect ground truth (GPR and rock core) data of mine roof anomalies. Numerical modeling serves as an alternative to generating ground truth data. This section presents the theory of numerical modeling of GPR signal.

Numerical modeling is a way of digitally representing real world problems or scenarios on the computer. Maxwell's equations $2.7-2.8$ in section 2.2 are partial differential equations (PDE) with the EM field vectors said to be continuous functions of time and position [19]. In order to model the wave propagation numerically and present 
them on a computer, these equations have to be converted from a continuous function into a discrete two-dimensional or three-dimensional function.

The FDTD technique, first presented by Kane Yee [21] in 1966, offers a direct solution for Maxwell's equations using the finite difference approximation. In Yee's original work on FDTD technique [21], he detailed what is presently known as the Yee cell, as taking a continuous model and discretizing it into cells consisting of a multidimensional grid-based model space [21] (Fig. 2.3). The condition for numerical effectiveness is the method at which electric $(\mathrm{E})$ and magnetic $(\mathrm{H})$ fields are determined. Thus, the spatial discretization of this grid-based model in $\Delta x, \Delta y, \Delta z$, and time discretization $\Delta \mathrm{t}$, play an important role in the FDTD methodology - since, the smaller the spatial and time discretization, the more representative of the finite difference approximation of the problem domain.

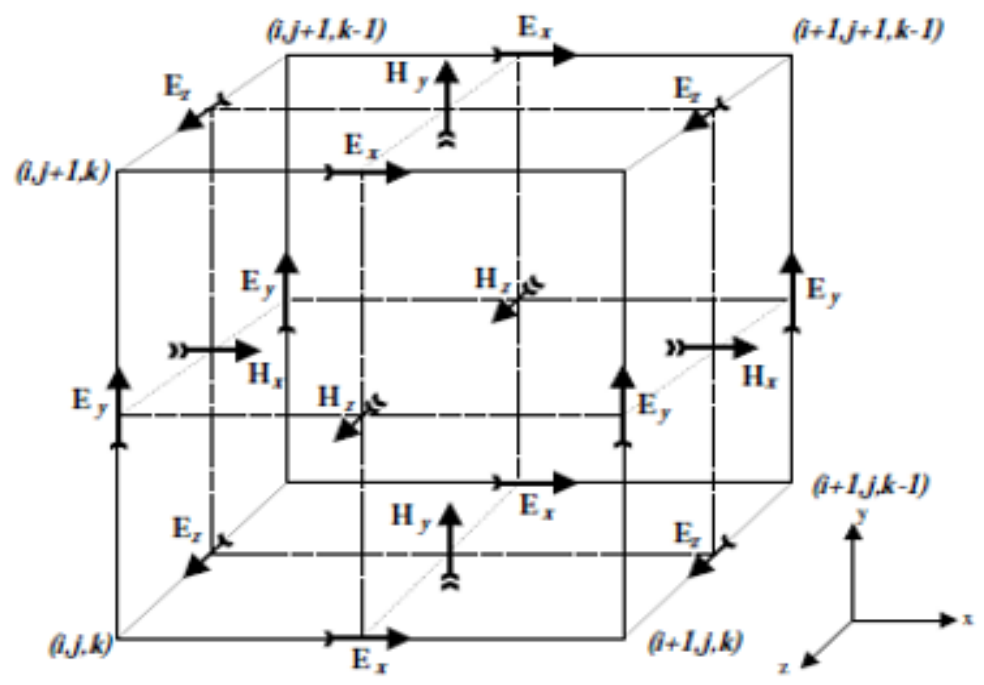

Fig. 2.3. FDTD Yee Cell indicating electric and magnetic field directions in 3D [21]. 
For a 2D model of a Cartesian coordinate system (x,y,z), the EM field vectors and model geometry have no variation with respect to the z-axis. The partial derivatives with respect to the z-axis in equations 2.7 and 2.8 will be zero, yielding [21]:

$$
\begin{aligned}
& \frac{\partial H_{x}}{\partial \mathrm{t}}=\frac{1}{\mu}\left(-\frac{\partial E_{z}}{\partial \mathrm{y}}-\rho^{\prime} H_{x}\right) \\
& \frac{\partial H_{y}}{\partial \mathrm{t}}=\frac{1}{\mu}\left(\frac{\partial E_{z}}{\partial \mathrm{x}}-\rho^{\prime} H_{y}\right) \\
& \frac{\partial E_{z}}{\partial \mathrm{t}}=\frac{1}{\epsilon}\left(\frac{\partial H_{y}}{\partial \mathrm{x}}-\frac{\partial H_{x}}{\partial \mathrm{y}}-\sigma E_{z}\right) \\
& \frac{\partial E_{x}}{\partial \mathrm{t}}=\frac{1}{\epsilon}\left(\frac{\partial H_{z}}{\partial \mathrm{y}}-\sigma E_{x}\right) \\
& \frac{\partial E_{y}}{\partial \mathrm{t}}=\frac{1}{\epsilon}\left(-\frac{\partial H_{z}}{\partial \mathrm{x}}-\sigma E_{y}\right) \\
& \frac{\partial H_{z}}{\partial \mathrm{t}}=\frac{1}{\mu}\left(\frac{\partial E_{x}}{\partial \mathrm{y}}-\frac{\partial y}{\partial \mathrm{x}}-\rho^{\prime} H_{z}\right)
\end{aligned}
$$

Equations $2.9-2.11$ and equations $2.12-2.14$ are now decoupled PDEs reflecting, respectively, the transverse magnetic (TM) mode and the transverse electric (TE) mode [21] in two-dimensions.

The solution of the numerical model is then derived in the time domain by utilizing the discretized Maxwell's curl equations (equations 2.9 - 2.14) implemented in each FDTD cell. The solution of the discrete model involves an iterative process. During each iteration, the EM fields propagates into the grid cells with each iteration representing an elapsed simulated time of $\Delta \mathrm{t}$ for a single trace (GPR signal). However, the estimation of 
$\Delta \mathrm{x}, \Delta \mathrm{y}, \Delta \mathrm{z}$, and $\Delta \mathrm{t}$ can't be assigned independently because to its restrictively steady numerical procedure, known as the CFL condition (also known as Courant, Freidrichs and Lewy condition) [35] given by the equation:

$$
\Delta t \leq \frac{1}{\sqrt[c]{\frac{1}{(\Delta x)^{2}+\frac{1}{(\Delta y)^{2}}+\frac{1}{(\Delta z)^{2}}}}}
$$

Where, $c$ is the speed of light. $\Delta t$ is a function of $\Delta \mathrm{x}, \Delta \mathrm{y}$ and $\Delta \mathrm{z}$ values.

In order to simulate the sub-surface layer of a potash mine, each Yee cell are assigned individual material properties (i.e. Dielectric constant, conductivity and permeability) and complex geometric structures can be included in the model that share the same properties. The FDTD solver introduces an absorbing boundary condition (ABC) to the boundaries of the generated model to avoid back reflections taking place when the EM wave reaches the boundary. With the utilization of suitable antenna source, all methods of EM propagation, dissipating, and target interactions are demonstrated and verifiable by the solver.

\subsection{EM wave propagation with subsurface materials}

It is necessary to understand the propagation of EM wave and the resulting response when it comes in contact with subsurface materials. These subsurface materials are buried targets that exhibits some electrical properties. The difference in electrical properties from the transmitting medium to the target creates the reflection signal. The following subsections describe the factors that affects a GPR signal generation. 


\subsubsection{Wave Propagation - Reflection, Refraction, Diffraction}

When an EM wave is dispersed and travelling through a medium encounters a subsurface with different material properties, the wave is partly reflected off the subsurface and partly reflected back to the receiver of the antenna. These discontinuities may be as a result of the interfaces between clay seams, void spaces, buried objects or landmines. The factors that affect the signal reflected depend on the nature (i.e. shape and geometry) and the material properties of the target.

As part of the EM wave reflects back to the receiver, the remaining part of the EM wave propagates through the subsurface based on the contrast between the two medium's dielectric constant by the principle of Snell's Law of Refraction [30] at a refracted angle with a change in velocity of the new propagating medium.

Diffraction occurs as a result of EM waves bending around targets. When targets are assumed to be point source, the diffraction of the wave produces a parabolic reflection as the wave tries to bend around it. The apex of the hyperbola is assumed to be the actual location of the point source or target.

\subsubsection{Material properties}

The propagation of EM wave in a medium is notably affected by the relative dielectric permittivity (dielectric constant) and the electrical conductivity of the medium.

\section{- Relative Dielectric Permittivity $\left(\varepsilon_{r}\right)$}

The time taken for an EM wave to travel from the transmitter to the subsurface and back to the receiver is dependent on the relative dielectric permittivity of the 
transmitting medium and the reflected medium. Different materials possess different dielectric permittivity depending on how much electrical charge it can store (table 2.1).

The amount of reflected energy obtained is dependent on the difference between the dielectric permittivity of the two materials as the EM wave travels from one material to the other material. Equation 2.16 is used in calculating the intensity of the reflected energy of two contrasting mediums [33].

$$
R E=I W \frac{\sqrt{\varepsilon_{r 1}}-\sqrt{\varepsilon_{r 2}}}{\sqrt{\varepsilon_{r 1}}+\sqrt{\varepsilon_{r 2}}}
$$

Where,

$R E=$ Intensity of the reflected energy

$I W=$ intensity of the incident wave

$\varepsilon_{r 1}$ and $\varepsilon_{r 2}=$ the relative dielectric permittivity for medium 1 and 2.

Table 2.1. Electrical properties of salt and related targets found in potash mine [31].

\begin{tabular}{|c|c|c|}
\hline Material & Dielectric constant & Attenuation db/m \\
\hline Pure salt and salt domes & $6-7$ & $0.004-0.02$ \\
\hline Saskatchewan halite & & $1-2$ \\
$(\mathrm{NaCl})$ & 5 & \\
& & \\
\hline
\end{tabular}




\begin{tabular}{|c|c|c|}
\hline $\begin{array}{c}\text { Saskatchewan sylvite } \\
(\mathrm{KCl})\end{array}$ & $5-5.5$ & $0.4-1.5$ \\
& & \\
\hline Saskatchewan clay seam & $5-6.5$ & $2-3$ \\
\hline Air (crack) & 1 & 0 \\
\hline Water & 80 & $0.01-100$ \\
\hline
\end{tabular}

\section{- Electrical Conductivity $(\sigma)$}

Another factor that affects the reflected GPR signal is the electrical conductivity of the medium. The 414 clay seam in potash mine is found to be lossy (i.e. possess some conductive property). The effect of increasing electrical conductivity makes EM wave penetration difficult. Multiples or "ring-down" in GPR data occurs when EM waves come upon conductive material or medium (i.e. metals) in its part. These metals when they come in contact with EM waves acts as an extra antenna transmitting EM waves thereby producing "ring-down" in GPR data. Equation (2.17) can be used to determine the electrical conductivity of a medium.

$$
\sigma=\frac{\sqrt{\varepsilon_{r}} \times \alpha}{1.69 \times 10^{3}}
$$

Where,

$\sigma=$ Electrical conductivity of the medium. 
$\alpha=$ attenuation of the signal

$\varepsilon_{r}=$ the relative dielectric permittivity of the medium.

\subsubsection{Attenuation}

As EM waves propagate through geological mediums, the signal suffers attenuation as a result of the uncertainty in the medium it encounters. The higher the frequency components of the signal, the more effect it has on the attenuation of the signal, resulting in the loss of resolution in radargram. Therefore, it is necessary to have less attenuation to produce quality GPR data.

Attenuation and radar scattering is also caused by poor ground coupling which can be as a result of improper contact of the GPR antenna with the roof (in potash mine) or ground surface. A way of overcoming this, is to ensure that the antenna is properly coupled with the ground surface and moving gradually to also accommodate uneven ground surface.

The interference of the radar signal by an electrical or magnetic field can reduce the energy absorption of the medium until they are fully dissipated or attenuated. The coefficient of attenuation is denoted as $\boldsymbol{\alpha}$.

\subsubsection{Limitations}

There is no doubt that GPR, being a non-destructive technique has yielded successful results with its application in the mining of potash. However, some of the limitations of GPR with its application to potash mine are listed below: 
1) The detection of the 414 clay seam becomes difficult when clay pockets come in contrast with the surrounding medium (salt). The depth, shape and length of the clay pocket affects the quality of the return signal.

2) Moist clay and slay water possess high conductivity which partially absorbs EM waves and makes GPR interpretation difficult.

3) GPR is difficult to apply to top ground layers that contain fastened rock bolts used as an extra support in mining because of its metallic properties. This produces "ring-down" in GPR data.

4) The dissemination of the clay seam (gradual change of materials having similar dielectric properties) makes it difficult to be detected by GPR.

5) GPR cannot conclusively specify the composition of the materials/soil without correlation and observation of rock-cores. Interpretations and estimates of the soil types can be made from GPR reflections by a geophysicist. Confirmation of soil types can be made by traditional borings (core data).

6) Ground coupling of antennas due to rough roof surface in mining room and material permittivity of salt, potash and clay affects the reflection of EM wave. The attenuation of the GPR signal cannot be easily determined by the amplitude of the reflected signal.

7) Depth penetration is an important factor that limits the use of GPR. Antenna design is necessary to determine how much depth penetration is required. In potash mine, a $1000 \mathrm{MHz}$ antenna is used for penetration of 3 meters. The detection of deeper layers is limited by the material properties and the antenna frequency used. 


\subsection{The GPR system design}

One important criteria in the design of a GPR equipment is its application area. GPR has a wide application range which determines the frequency range and type of antenna polarization suitable for its application. Based on its application area, the GPR must be able to achieve the depth information of its target, satisfactory spatial resolution, adequate signal to noise ratio (SNR) and signal to clutter ratio (SCR). The performance of a GPR signal is dependent on the following factors: range, velocity of propagation, clutter, depth resolution and plan resolution.

\subsubsection{Range}

The range of a GPR is represented by the path loss of its signal. There are three primary contributors to this: material loss, dispersion loss, and target reflection loss. As the signal travels from the transmitter to the receiver, losses are encountered through the

propagation path. For a specific distance, the total path loss is governed by Equation 2.18 [8]:

$$
L_{T}=L_{e}+L_{m}+L_{t 1}+L_{t 2}+L_{a}+L_{s}+L_{s c}
$$

Where,

$L_{e}=$ antenna efficiency loss

$L_{m}=$ antenna mismatch loss

$L_{t 1}=$ transmission loss from air to material

$L_{t 2}=$ retransmission loss from material to air

$L_{a}=$ attenuation loss from material

$L_{s}=$ antenna spreading loss 
$L_{S C}=$ target scattering loss

\subsubsection{Velocity propagation}

In other to obtain the depth information of a target, the velocity of the EM propagation is first derived. If the material is isotropic and homogeneous, the relative propagation velocity $v_{r}$ can be calculated using Equation 2.19

$$
v_{r}=\frac{c}{\sqrt{\varepsilon_{r}}} m s^{-1}
$$

The resulting depth information is derived using Equation 2.20

$$
d=v_{r} \frac{t}{2} m
$$

Where,

$\varepsilon_{r}=$ relative dielectric constant

$t=$ two way travel time (TWTT) recorded from the transmitter back to the receiver.

The velocity of propagation varies for different materials with different dielectric constant. Fig. 2.4 shows the relationship between the relative permittivity and the propagating velocity. With increasing dielectric constant, the propagating velocity and the wave length decreases. Eq. (2.21) shows the relationship of the wave length and the propagating velocity.

$$
\text { wavelength }(\lambda)=\frac{v_{r}}{f} m
$$




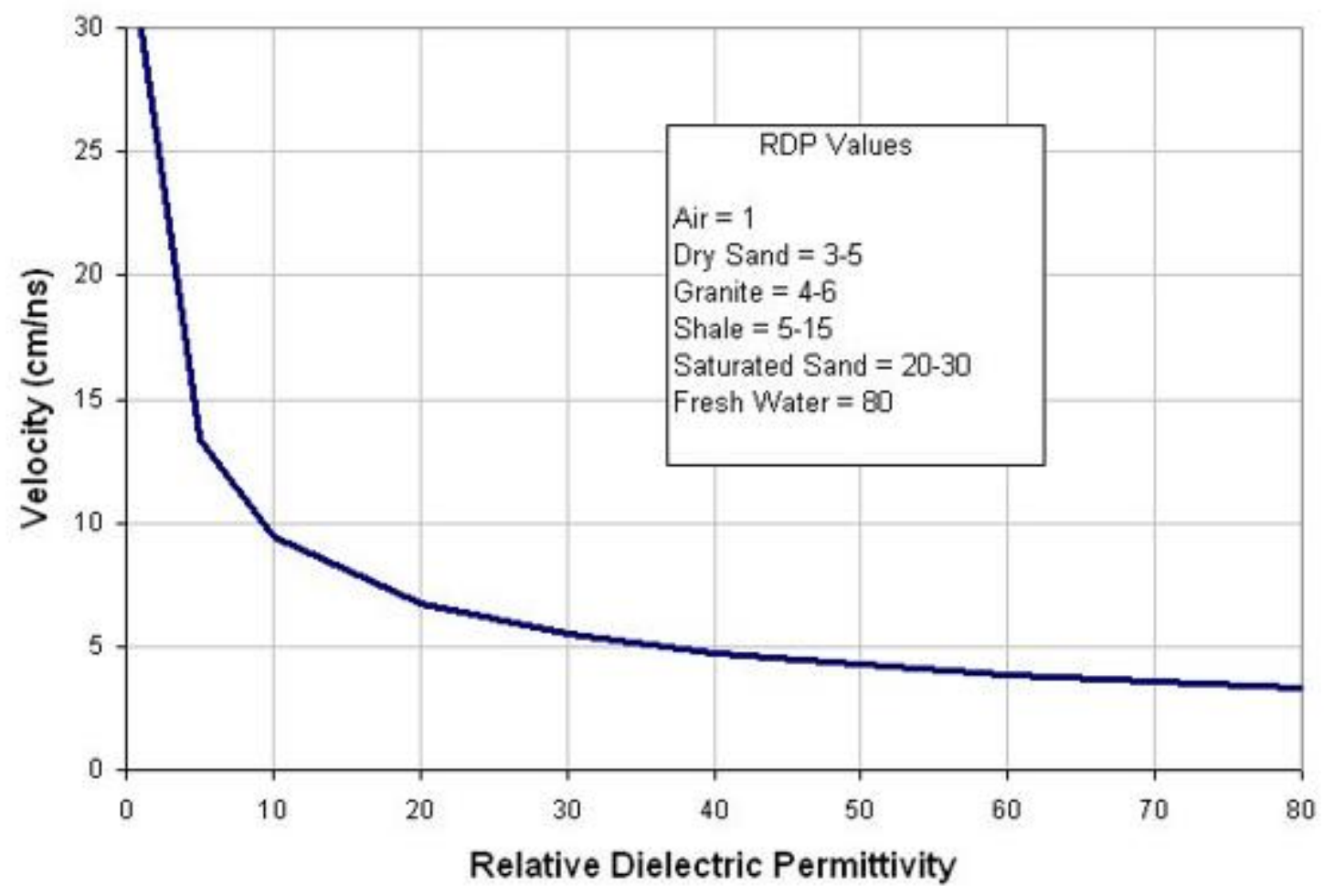

Fig. 2.4. Velocity propagation against relative dielectric permittivity [8].

\subsubsection{Clutter}

Clutter can be described as noise present in a GPR signal. It possesses similar characteristics as the actual target wavelet but is not related to the target signal. Numerous reflections between the antenna and the ground surface (ground coupling) can give rise to clutter. Also, the breakthrough between the transmitter and receiver produces clutter. These clutter varies according to the antenna configuration. Clutter is often visible at shorter range time and decreases over a long range of time. 


\subsubsection{Depth resolution}

Larger wavelengths are required to distinguish between multiple targets. Whereas for a single interface, it is easy to estimate the depth resolution from the time record of the signal. The wavelength of the received signal could easily compare to that of the transmitting signal [8]. The power spectrum is used to determine the wavelength of the received signal [8]. Lower frequency antennas provide more depth information compared to higher frequency antennas.

\subsubsection{Plane resolution}

GPR data plan resolution is necessary where multiple targets are buried with the same depth. A higher bandwidth antenna is required for location accuracy. The plan resolution is a function of the characteristics of antenna deployed and the signal processing incorporated. A higher frequency is characterized by a shorter wavelength with a better resolution of target while a lower frequency is characterized by a longer wavelength and poor resolution.

\subsection{The GPR system}

The GPR machine consist of four major components: a control unit, a transmitting antenna, a receiving antenna, and a portable computer.

The interaction between the transmitting and receiving antenna of the GPR with a buried target is seen in fig. 2.5. An EM wave is generated by the control unit and emitted into the ground through the transmitting antenna (Tx). The signal spreads in the medium by means of diffraction but the EM waves will be dispersed in all directions if the signal 
hits a target (buried object) or subsurface (which can be an interface between two geological layers), or the return signal is intercepted by the receiver of the antenna $(\mathrm{Rx})$. The dispersion of the signal is dependent on the refraction of the EM wave, which helps identify the target. Most times, the transmitting and receiving antenna are located in the same location with a separation distance to avoid reverberation. The reflected signal is then displayed on the computer screen for the operator.

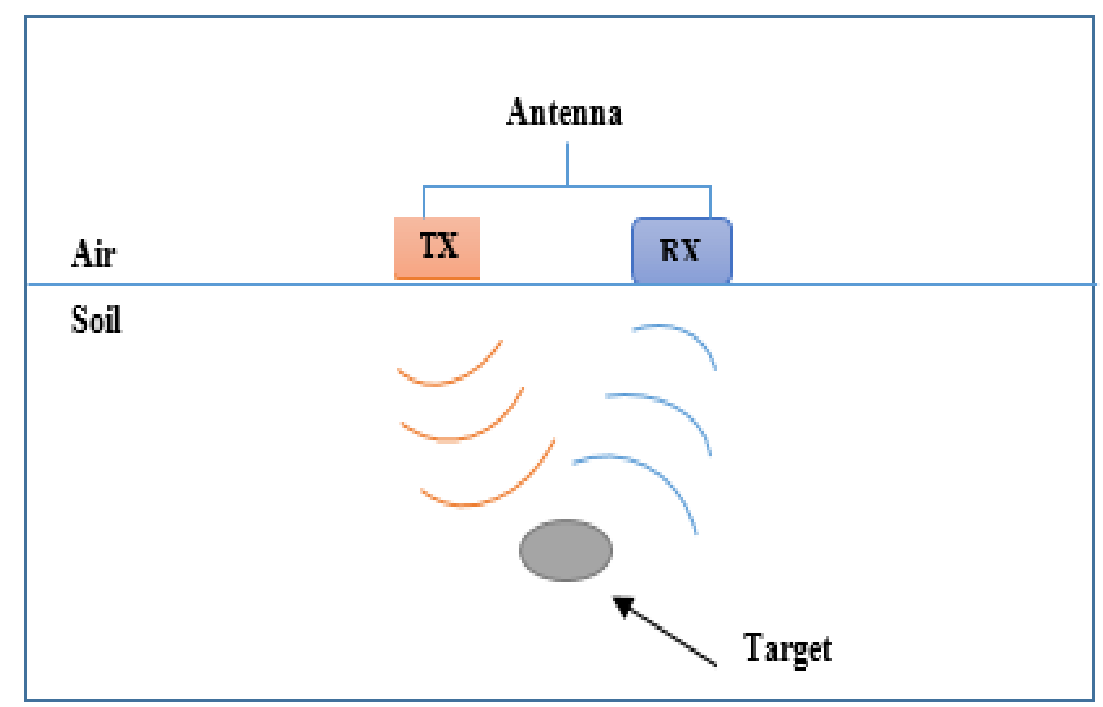

Fig. 2.5. Interaction between the transmitter and receiver of an antenna.

Fig. 2.6 shows the typical setup and main components of a GPR System made-up in BackTrak [23] for mine roof assessment. The system is a $1000 \mathrm{MHz}$ PulseEkko GPR system designed by Sensors \& Software for potash mine application. The GPR system in the picture uses an odometer that stores the distances for the measured data. This is important because the GPR is moved by a human operator and is consequently, not moved with a constant velocity. At every step reading of the odometer, EM wave is emitted into 
the ground and back, multiple traces are stacked together and averaged to determine the final GPR trace.

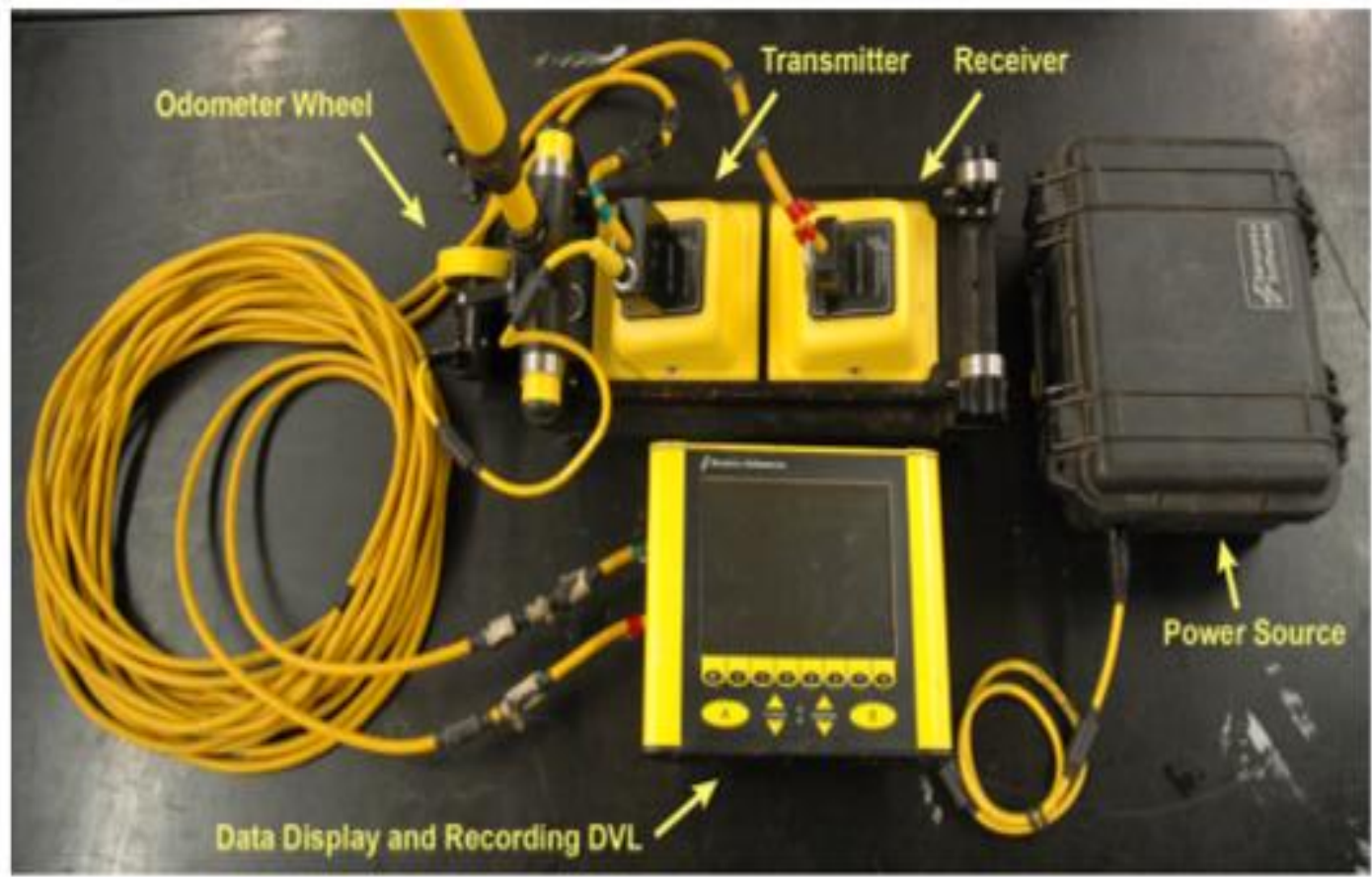

Fig. 2.6. $\quad$ Sensors \& Software 1000 MHz PulseEkko GPR [23].

The GPR operates in the ground which is more complicated than air due to heterogeneous, anisotropic, and/or magnetic soils. The GPR works with static targets and the interpretation is usually performed in post processing of the raw data.

There are different types of antennas with a range of frequencies available and the required band of frequencies depends on the application. Lower frequency antennas better penetrate structures but at the same time, provide less resolution of features in comparison to high frequency antennas. 


\subsection{GPR data acquisition}

A general GPR survey is implemented by placing a GPR device through a review line to get a GPR profile of the ground or roof (potash mine). The operator of the GPR machine performs an inspection either by manually attaching a wheel with encoders or towing the GPR device with a vehicle. The frequency of the antenna for a GPR survey is dependent on the goal of study and point of interest in the study region. The geological stratigraphy of the underground potash mine is also a very supportive tool for the GPR operator while boring, as EM waves performs poorly due to the material properties of clay soil and depth penetration is diminished.

The common offset (CO) and common mid-point (CMP) (see Fig. 2.7 and 2.8 respectively) are the two most common GPR data acquisition modes of placing the transmitting and receiving antennas during a GPR survey. The GPR system used in the potash mine makes use of the common offset mode in which the transmitting antenna is located at a fixed distance from the receiving antenna and is coupled together as a unit. 


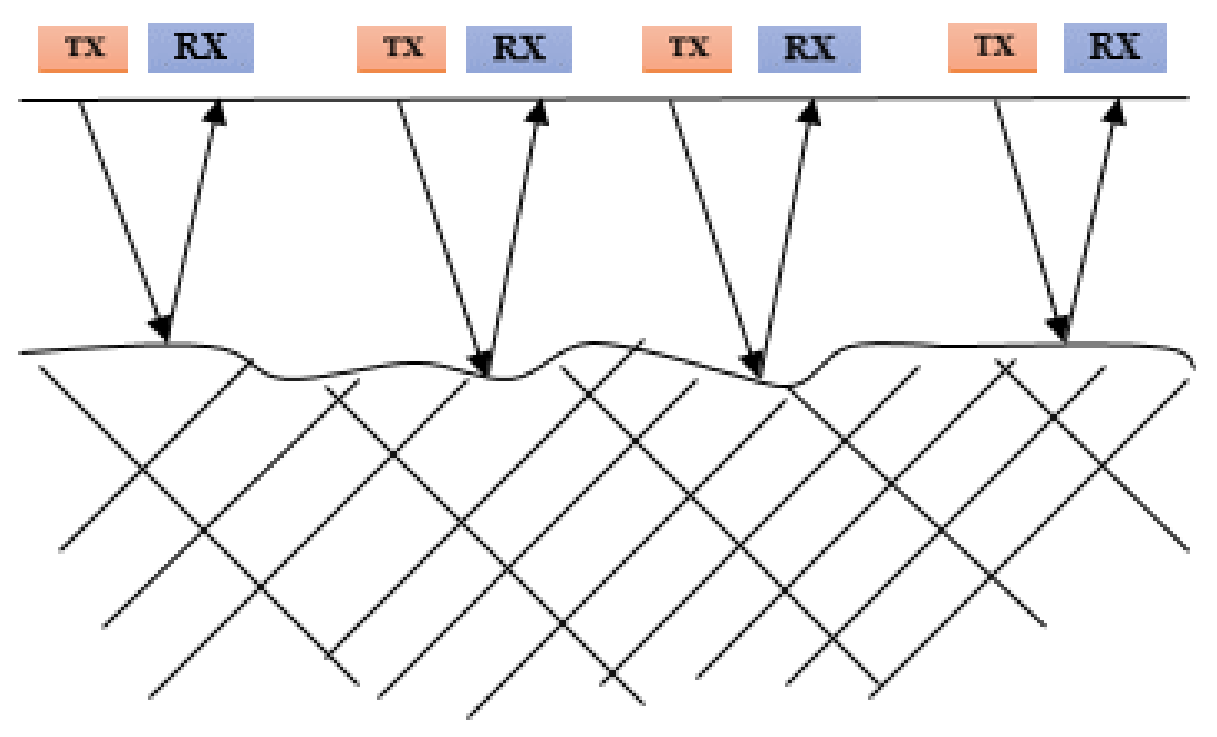

Fig. 2.7. A schematic representation of a common offset method.

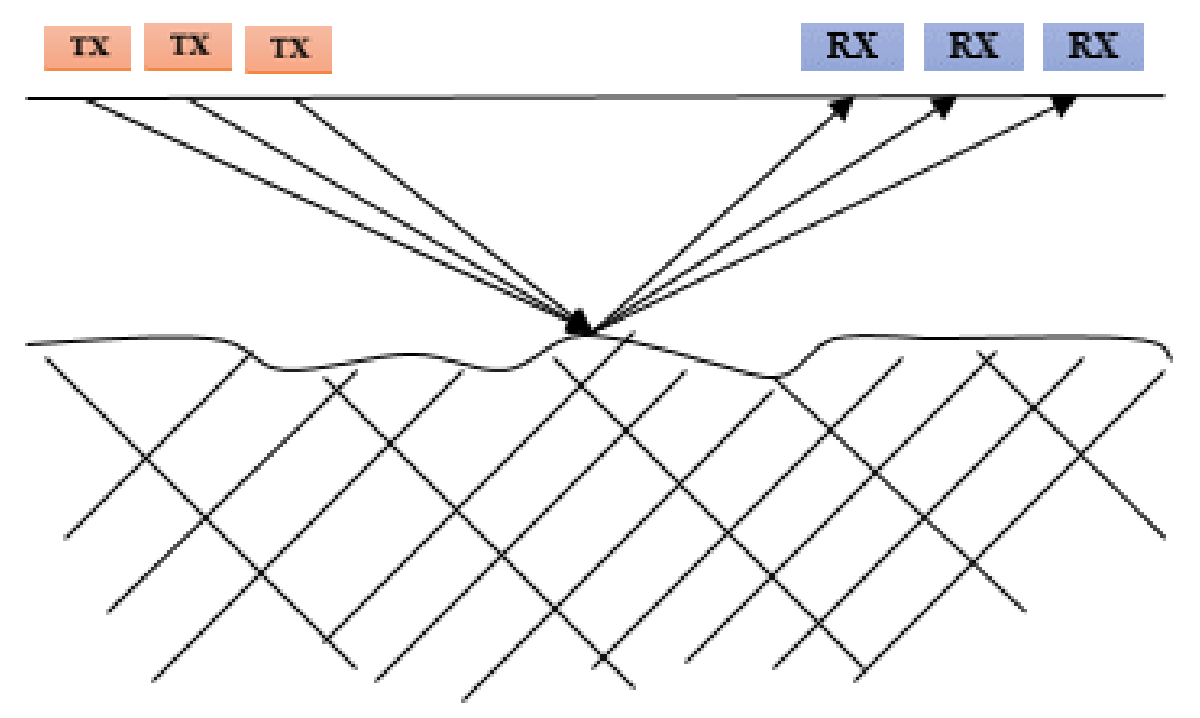

Fig. 2.8. A schematic representation of a common midpoint (CMP) survey method. 


\subsection{GPR data display}

The purpose of a GPR data display is essentially to give a visual representation of a GPR survey and show region of interest expected to be seen in the data and the target location in their appropriate spatial positions [14]. GPR data display is vital in interpreting GPR data.

A common GPR data display technique is using a colour intensity to represent the variation in field strength or amplitude intensity of the received GPR signal. Another method is the use of the wiggle plot which is commonly used in seismic data. The wiggle plot is a collection of single traces with no colour intensity.

The following subsections briefly explains the terminologies used in displaying GPR data [12], [13], [14], and [15].

\subsubsection{A-scan}

A-scan is a 1-D data representation of a GPR data with a field strength (amplitude) against time plot that is recorded by a single collection of a GPR signal with the antennas at a given position (x, y). Fig 2.9 shows a typical A-scan. 


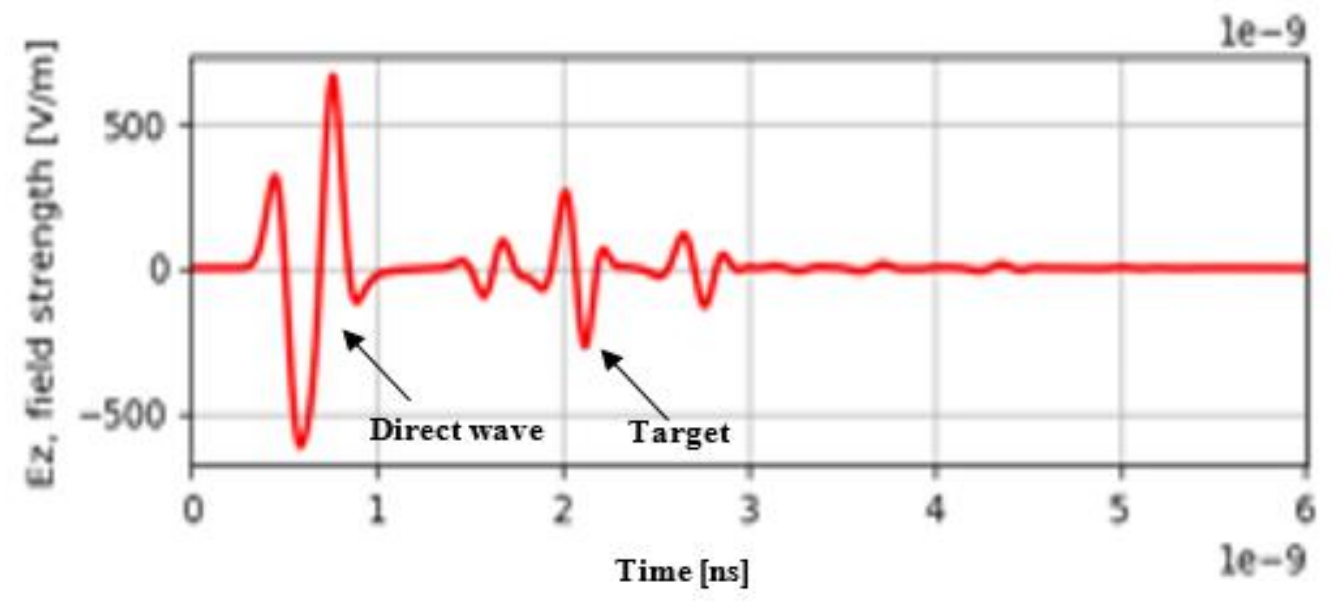

Fig. 2.9. A-scan of a GPR signal.

\subsubsection{B-scan}

While moving a GPR device along a line (x-axis), multiple GPR traces (A-scan) are recorded, the single traces are combined together to form a 2-D (two dimensional) data set called a B-scan. The 2-D image represents vertical slices of single GPR traces of the field strength against time. Fig. 2.10 shows the wiggle plot of multiple A-scan with specified target combined to form a B-scan (radargram).
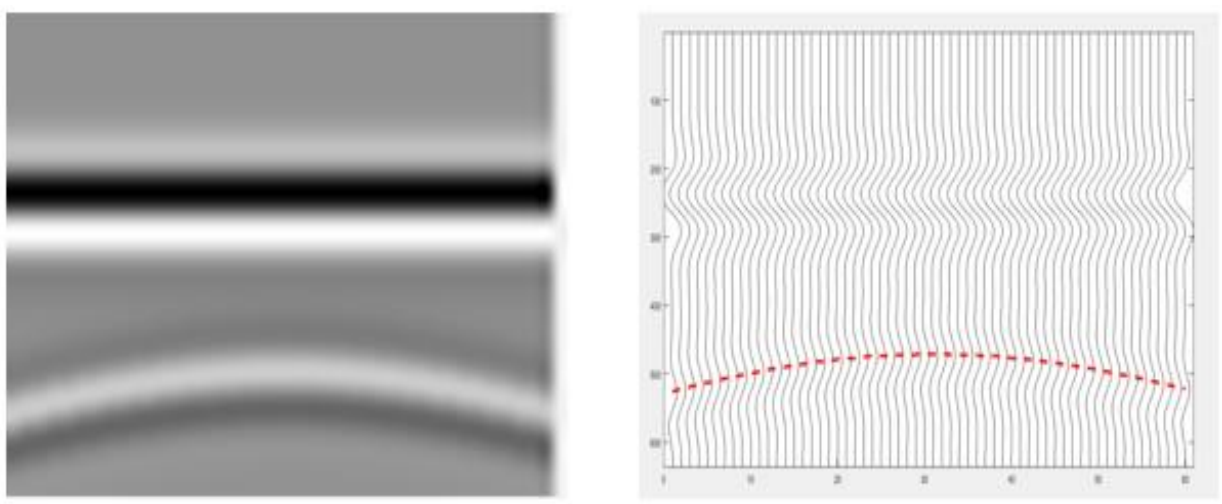

Fig. 2.10. Generation of a B-scan. The wiggle plot on the right shows the combination of series of A-scan with the profile of the target. 


\subsubsection{C-scan}

C-scan is a 3-D data representation of GPR signal gotten by stacking B-scans or, multiple parallel B scans along the plane. Acquiring a decent 3-D image is important for specific target identification. The targets are often simpler to distinguish and interpret on a C-scan than a B-scan. Fig. 2.11 shows the stacking of B-scans to form a C-scan.

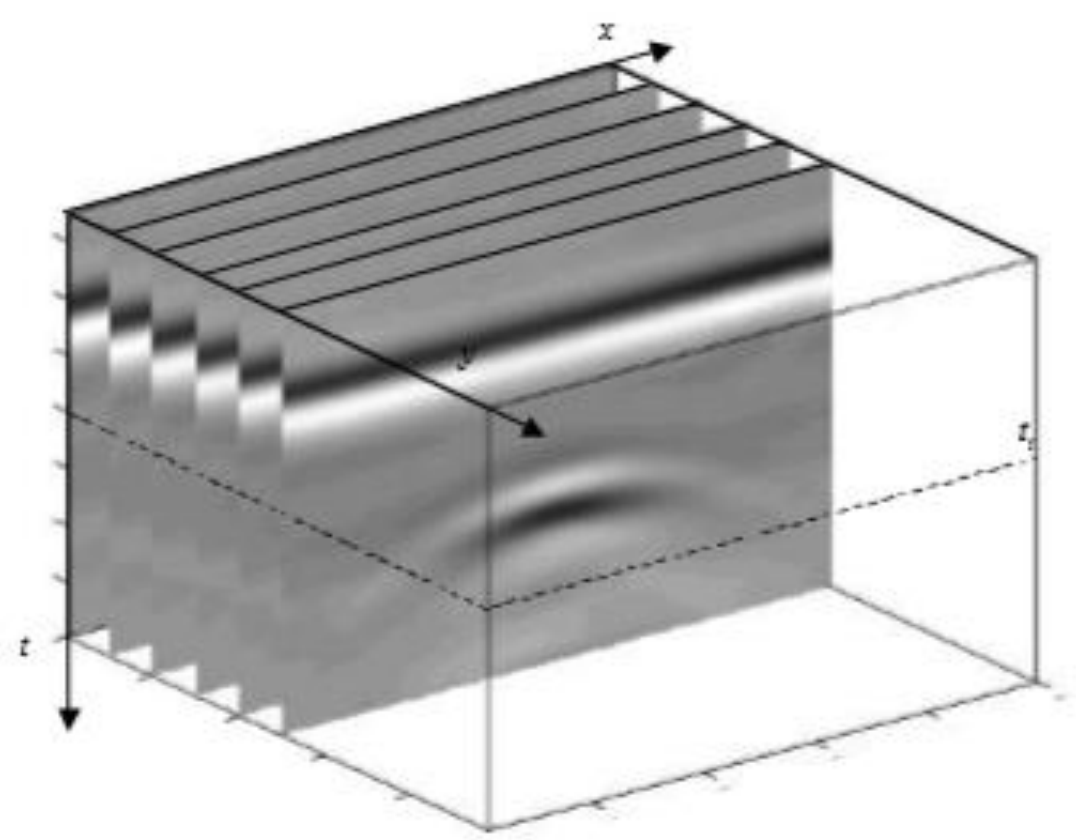

Fig. 2.11. Combination of several B-scan to form a C-scan. 


\subsection{GPR data processing}

In order to generate GPR signals with adequate signal to noise ratio (SNR) to extract meaningful information from the radargram, GPR data processing is implemented. The data processing helps to reduce clutter or eliminated unwanted noise in the data set. The GPR processing is carried out on raw data before being displayed for the operator. General processing applied to GPR data are trace editing, filtering, or data correction [10] [16]. Some of the processing applied to potash mine GPR data are briefly discussed below.

\subsubsection{Time-zero correction}

Before processing is applied to the GPR data, the traces are adjusted to a common time-zero position. This ensures that all reflections are correctly aligned by setting the airwave/direct wave of the trace at the first break point (or the first negative lobe) to a particular time-zero position [37]. Time-zero correction is usually the first processing applied to a GPR data.

\subsubsection{Dewow}

Dewowing is a processing technique used to eliminate low frequency components from a GPR data by implementing a high-pass filter on the data to reduce the noise level to a zero mean [38].

\subsubsection{Background subtraction}

Background subtraction is the removal of the background signal from the GPR data that is present at the beginning of a radar signal known as the direct wave. This part of the signal is considered as unwanted noise (also called clutter) produced by antenna ringing 
caused by multiple reflections occurring between the ground surface and the antenna (ground coupling) [8] [36].

\subsubsection{Gain}

Gain is a basic processing technique which helps to enhance the display of the GPR data by suppressing noise and enhancing the target reflection. This step is necessary in order to equalize the amplitude of the emitted wave, which suffers a significant attenuation along the medium [38]. Automatic Gain Control (AGC) and Spreading and Exponential Compensation (SEC) [18] are the two most common types of gain applied to GPR data $[18]$

\subsubsection{Migration}

Migration is a processing technique which enhances GPR data by properly placing dipping reflectors to their true position and produce better radargram representation of the GPR profile [17] [18].

\subsection{Application of GPR}

GPR technique has proven to be a successful method used for non-destructive testing and evaluation with a wide scope of applications. GPR has been used in mapping of rock depths, detecting voids and cracks, archaeological investigation, detection of buried objects such as pipes, cables, etc. Typical applications are listed in table 2.2. 
Table 2.2. Typical Applications of Ground Penetrating Radar [10]

\begin{tabular}{|l|l|}
\hline Field of study & Application area \\
\hline Civil Engineering & $\begin{array}{l}\text { Buried pipe and cable mapping } \\
\text { Pipe leak detection } \\
\text { Void detection } \\
\text { Pavement thickness } \\
\text { Bar reinforcement }\end{array}$ \\
\hline Geotechnical & $\begin{array}{l}\text { Potash mining } \\
\text { Coal mining } \\
\text { Peat profiling } \\
\text { Detection of UXO } \\
\text { Investigation }\end{array}$ \\
$\begin{array}{l}\text { Metallic and Non-metallic landmines detection } \\
\text { Ice thickness profiling } \\
\text { Sinkhole prediction } \\
\text { Geological strata profiling } \\
\text { Hazardous waste mapping } \\
\text { Road pavement thickness and assessment } \\
\text { Railroad bed profiling and tie evaluation } \\
\text { Archaeological prospecting } \\
\text { Runway integrity testing and evaluation }\end{array}$ \\
\hline
\end{tabular}




\subsection{Auto-Picking Techniques}

During mining, an automated process of tracking the 414 clay seam is used to provide the operator of the mining machine with a real-time notification of the salt-back thickness. Two auto-picking techniques are reviewed in this thesis that are applicable to potash mines.

\subsubsection{BackTrak}

BackTrak is a commercial GPR system designed by Sensors \& Software [23], in partnership with Nutrien Limited, specifically for use in underground potash mines. It contains an auto-picking algorithm used for potash mine roof assessment and interpreting GPR data currently applicable to potash mine. A desktop version of the BackTrak software was provided by Sensors \& Software for this research. Once provided with GPR data (actual or synthetic), the software would generate a sequence of 'picks' and interpreted positions of the clay seam.

\subsubsection{Clustered Ratio Derivative (CRD)}

The second auto-picking technique applied is the CRD algorithm [24]. The CRD algorithm is based on a modification of the well-established STA/LTA algorithm used for picking first-breaks in vibroseis / seismic data [25] [26]. The CRD algorithm makes use

of three techniques that can be grouped and used on the A-scan or B-scan of a GPR data as seen in Fig. 2.12. The algorithm's three main components are: 
- Ratio: Short Term Average / Delayed Term Average (STA/DTA) and Derivatives.

- Density-based spatial clustering of applications with noise (DBSCAN)

- On-line Outlier Detection and cleaning.

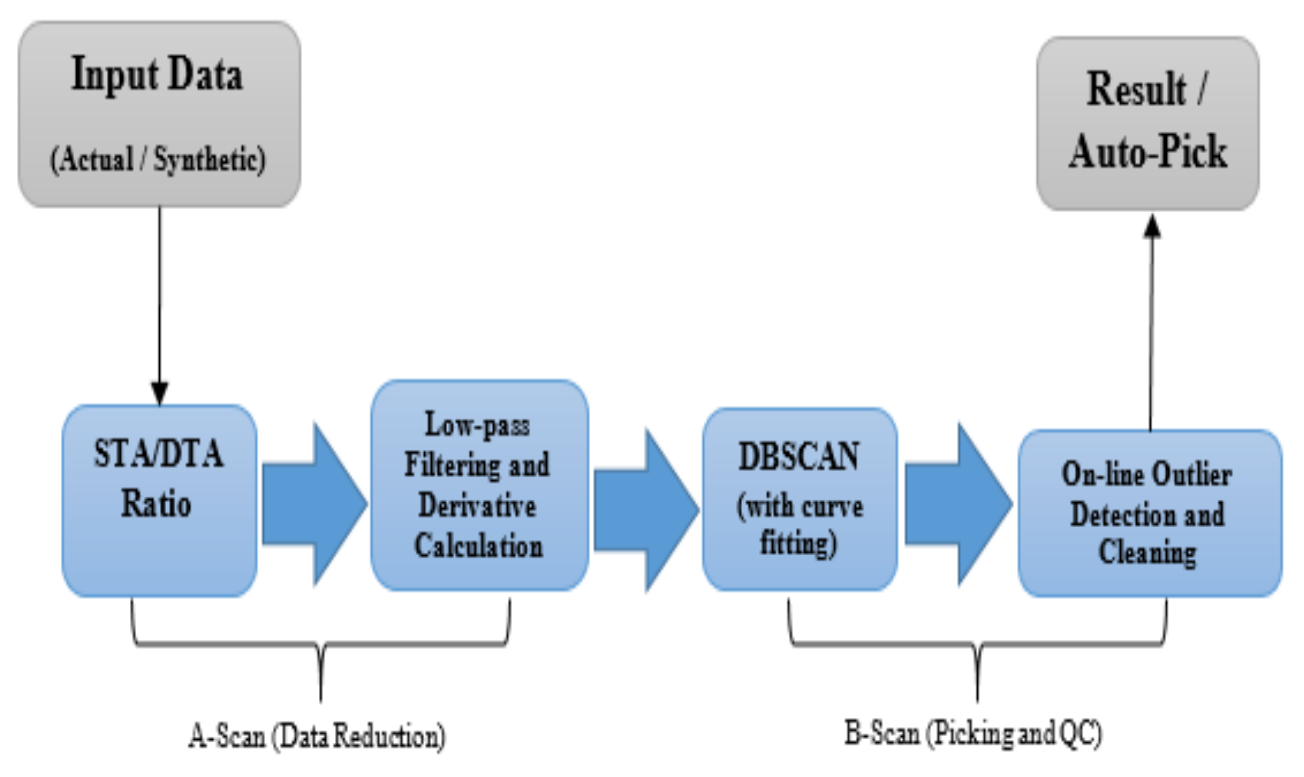

Fig. 2.12. A flowchart of the CRD algorithm process on GPR Data.

The CRD algorithm was implemented on the actual mine data and compared picked result done by a geophysicist (ground truth). The standard deviation (SD), percentiles, and mean were used to assess the performance of the CRD algorithm.

Table 2.3 shows the result of the CRD algorithm applied to the mine data, which shows an average $97 \%$ accuracy of the auto-picking algorithm. The percentiles show the minimum accuracy that would be expected from 90 and 95 percent of the data set. 
Table 2.3. Statistical result on actual data [24]

\begin{tabular}{|c|c|c|c|c|c|c|}
\hline & & $\begin{array}{c}546 \mathrm{~W} \\
\text { Cory } \\
(1020 \mathrm{~m})\end{array}$ & $\begin{array}{c}452 \mathrm{~N} \\
\text { Cory } \\
(184 \mathrm{~m})\end{array}$ & $\begin{array}{c}513 \mathrm{~N} \\
\text { Cory } \\
(1550 \mathrm{~m})\end{array}$ & $\begin{array}{c}\text { Nlle } \\
\text { Allan } \\
(1460 \mathrm{~m})\end{array}$ & $\begin{array}{l}\text { PEC351 } \\
\text { Lanigan } \\
\text { (500m) }\end{array}$ \\
\hline \multirow{4}{*}{$\begin{array}{c}\text { Accuracy } \\
\text { with respect } \\
\text { to actulal } \\
\text { distance (m) } \\
\% \\
\text { [Part A] }\end{array}$} & 90 percentile & 97.87 & 97.26 & 95.73 & 93.15 & 73.36 \\
\hline & 95 percentile & 96.97 & 95.55 & 91.42 & 88.48 & 66.58 \\
\hline & Mean accuracy & 98.96 & 98.56 & 97.15 & 97.02 & 88.35 \\
\hline & $\begin{array}{l}\text { Standard } \\
\text { Deviation }\end{array}$ & 1.18 & 3.84 & 6.41 & 5.77 & 10.45 \\
\hline
\end{tabular}




\section{Chapter 3.}

\section{Methodology}

This section discusses how GPR data used for this thesis is acquired, analysed and interpreted by a trained geophysicist which serves as the ground truth for the simulated data. The ground truth is then used to generate synthetic GPR signals (A-scan and B-scan) and compared with the actual data. Also, a method of defining 'stray clay' is further injected into the numerical model to create confounding problems for auto-picking algorithms.

\subsection{Survey location and geological setting}

The GPR survey used for this thesis was conducted at the Cory Mine Division, operated by Nutrien Limited. Nutrien describes mining activity at this location as being done at the Upper Patience Lake Member (A-zone) which is 1000 meters below the surface [2]. Fig.1.1 describes the stratigraphy of the A-zone as consisting of several geological markers representing clay seams present in the mine. The borer operator uses the 413 clay seam as a cutting marker. Stratigraphy above the 413 clay seam consists of about a meter of salt followed by the 414 clay seam (a dominant clay seam). The absence of cohesion in clay seams poses a serious safety concern in potash mines as they cause weakness in the salt-back. Also, bifurcation of the 414 clay seam creates clay pockets within the salt-back which can contribute to a salt-back failure. These clay pockets are referred to as 'stray clays' for the purposes of this thesis. 


\subsection{Data acquisition}

\subsubsection{Actual Data}

For this research, GPR data was collected using an acquisition system called BackTrak - a specific potash mine software. . This software was co-developed by Sensors \& Software and Nutrien Limited specifically for use on mining machines in potash mines. Table 3.1 shows the parameters used for the data acquisition.

Table 3.1. Data acquisition parameters

\begin{tabular}{|l|l|}
\hline Data Machine & Kubota ATV (GPR-RTV) [2] \\
\hline Software & BackTrak \\
\hline Antenna & $1000 \mathrm{MHz}$ Noggin Gold Antenna \\
\hline Depth & 3 meters \\
\hline Velocity & $0.120 \mathrm{~m} / \mathrm{ns}$ \\
\hline Stack & DynaQ Stacking \\
\hline Station interval & 0.05 meters \\
\hline
\end{tabular}

BackTrak utilizes a ground coupled 1000MHz Noggin GPR antenna mounted on a Kubota ATV [2] (Fig. 3.1). The installation and implementation of BackTrak for roof assessment is discussed in [2]. During the GPR survey, the raw data collected is then processed in EKKO_Project software [6] (Sensors \& Software Inc.) using the following techniques: Data repositioning, first break redatuming (20\%), dewow, background subtraction (201 traces) and band pass filtering [2]. Figure 3.2 shows the resulting GPR line survey collected with the Kubota ATV. 


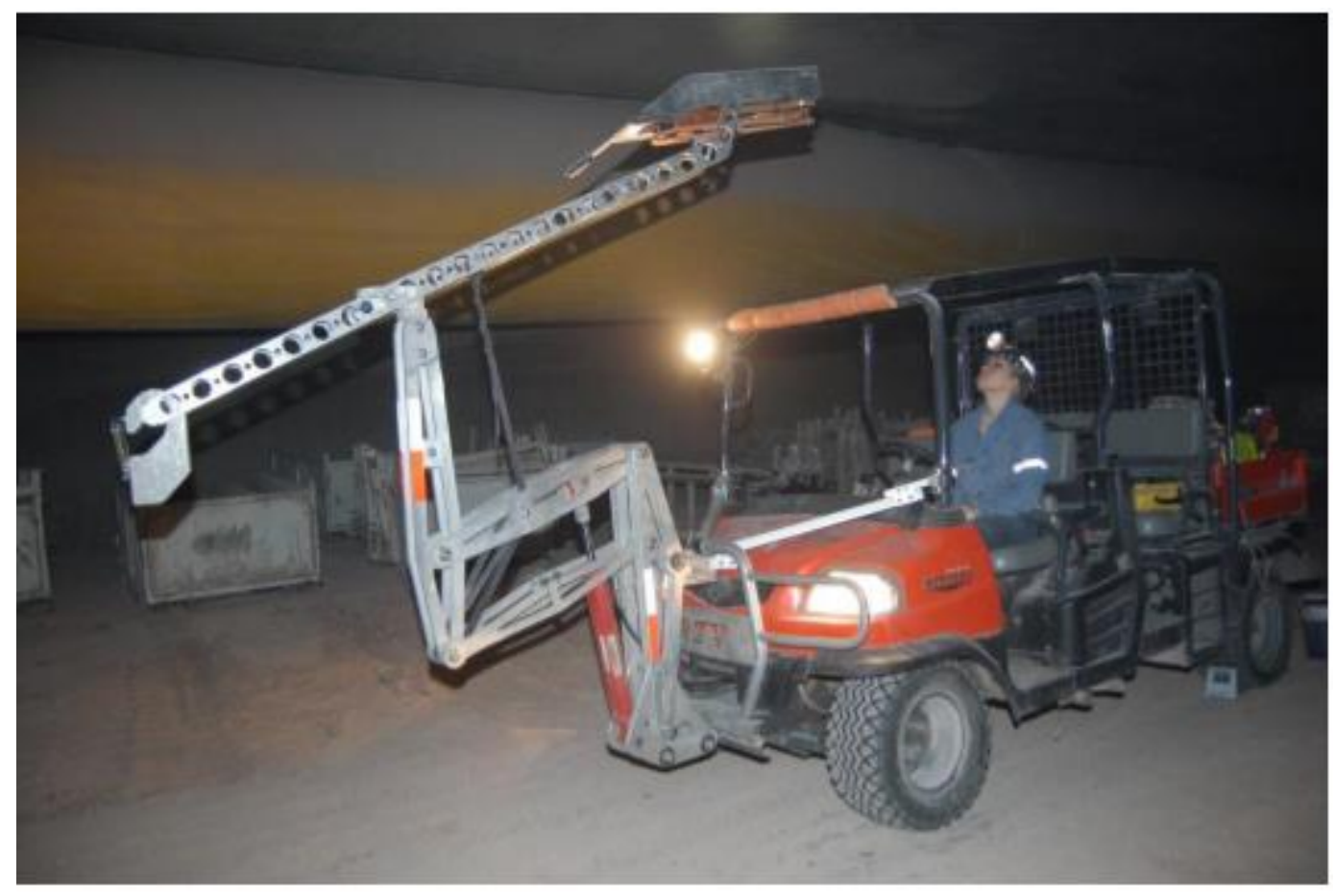

Fig. 3.1. Kubota ATV used for GPR survey.

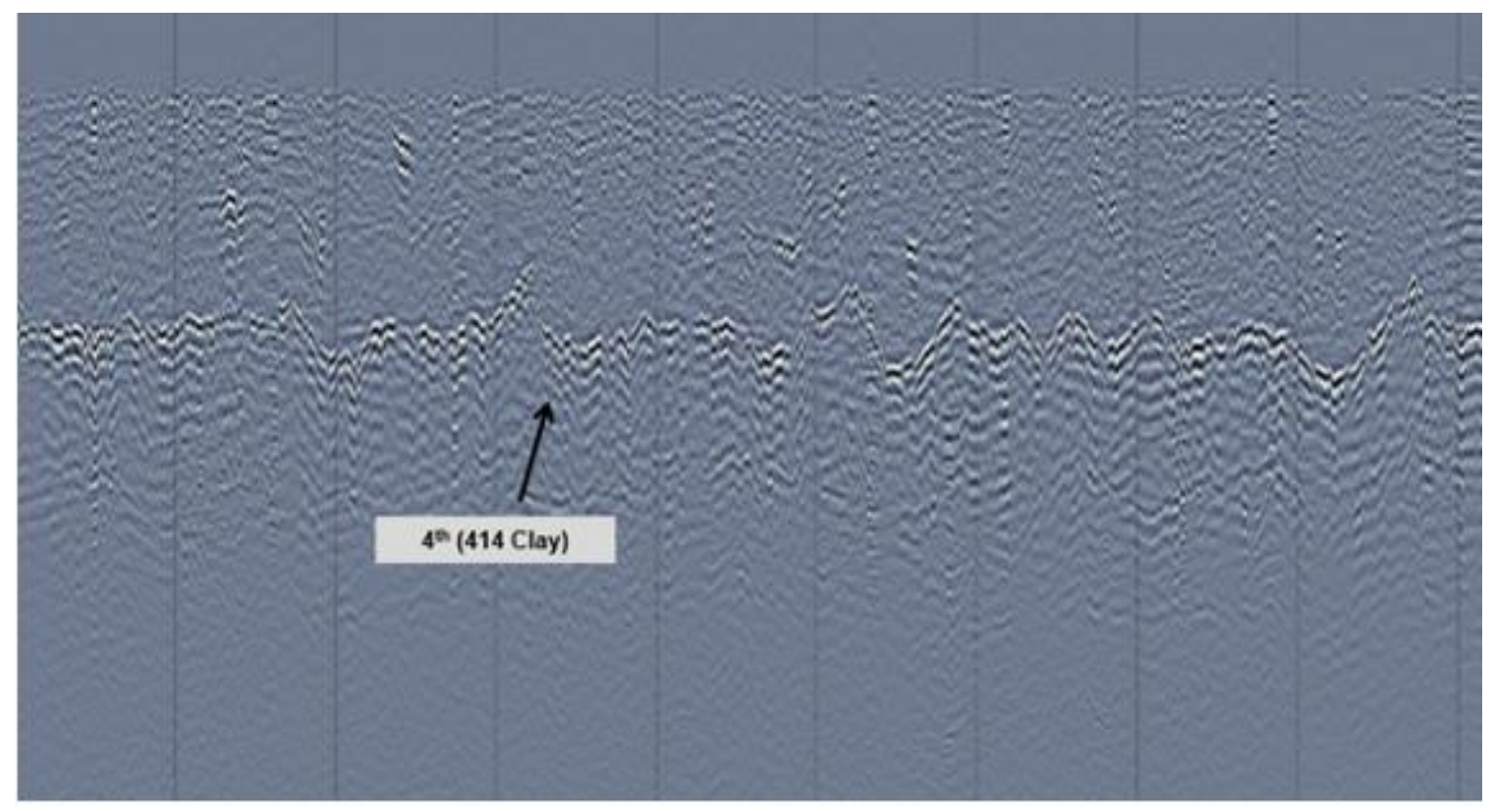

Fig. 3.2. B-scan of the GPR survey. 
Fig. 3.3 presents a typical example of GPR data (B-scan) which was collected using this system and interpreted by a trained geophysicist to show the position of the 414 clay seam. This GPR data was provided by Nutrien Limited. While there are examples of 'stray clays' in this data set (as seen in Fig. 3.3), the geophysicist is clearly able to distinguish between the actual 414 clay seam and the stray clays. It is important to note that the presentation of a typical GPR data is inverted from the actual geology. This means that the roof of the mining room is at the top of the image while the mine geology (including the 414 clay seam) is shown below the roof.

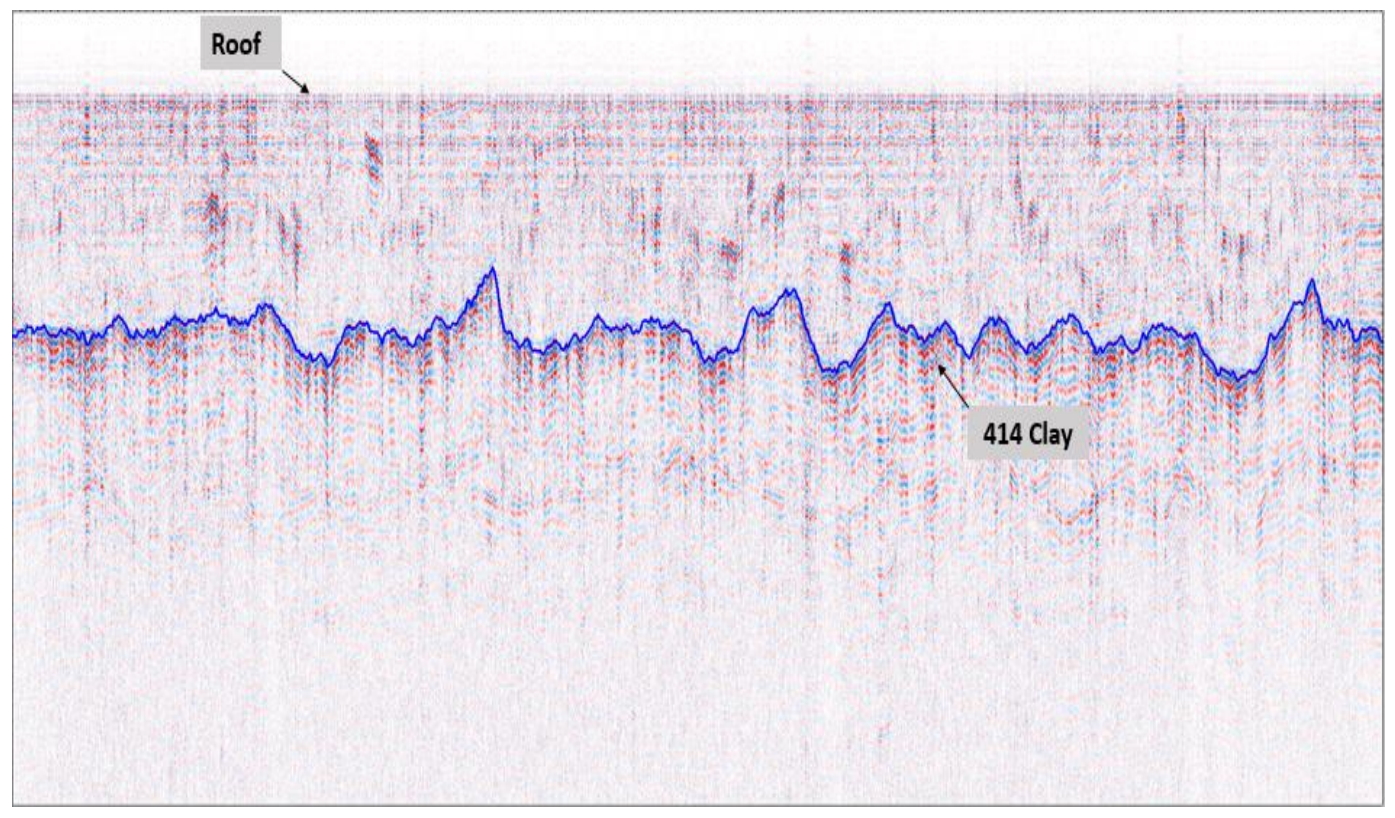

Fig. 3.3. Nutrien Geophysicist's interpretation of 414 Clay seam from GPR data collected using a Kubota (GPR-RTV). 


\subsubsection{Numerical data}

Numerical data was generated by integrating a text file of the geophysicist interpretation of the depth of the 414 Clay seam from an actual mine data (Fig. 3.3) is imported into gprMax. This allowed the creation of representative numerical models consisting of 2D data sets of simulated GPR response signals of an actual potash mining room roof.

The gprMax software adopts the FDTD technique to solve Maxwell's equations by representing EM wave propagation through materials with different layers of thickness and electrical properties. The FDTD method was first introduced by Kane Yee [9] who formulated the well-known Yee cell. The Yee cell is comprised of a three-dimensional (3D) grid representation of the 3D space over which the GPR response signals are calculated. The model is developed by transforming a continuous world into a discretized model with $\Delta \mathrm{x}, \Delta \mathrm{y}$ and $\Delta \mathrm{z}$ components. The strength of the electrical and magnetic fields at these fixed points is sequentially computed and analyzed. In this work, a spatial resolution (level of discretization) for $\Delta \mathrm{x}, \Delta \mathrm{y}$ and $\Delta \mathrm{z}$ is set to equal $0.002 \mathrm{~m}$ respectively. The frequency of the antenna used is a $1000 \mathrm{MHz}$ modelled as a point source in gprMax. The antenna is sampled with a step size of $0.05 \mathrm{~m}$ along the $\mathrm{x}$-axis. The domain dimension along the $\mathrm{x}$-axis becomes the number of sample points multiplied by the step size to get the distance in meters. Two materials present in the created models were salt (transmitting medium) and clay (reflecting medium) as seen in Fig. 4.1a of section 4.1. In gprMax, salt and clay are defined by the following material characteristics: dielectric constant $\left(\varepsilon_{r}\right)$, 
conductivity $(\sigma)$, relative magnetic permeability $\left(\mu_{r}\right)$, and magnetic loss $\left(\sigma_{*}\right)$. These are shown in Table 4.2 below.

Table 3.2. Model parameters.

\begin{tabular}{|l|l|}
\hline Domain size & $27.2 \mathrm{~m} \times 1.5 \mathrm{~m} \times 0.002 \mathrm{~m}$ \\
\hline Time window & $30 \mathrm{~ns}$ \\
\hline Waveform & BackTrak \\
\hline Frequency & $1000 \mathrm{MHz}$ \\
\hline Antenna type & Point dipole \\
\hline Step size & $0.05 \mathrm{~m}$ \\
\hline Medium 1 = Salt & $\varepsilon_{r}=2.7, \sigma=0, \mu_{r}=1, \sigma_{*}=0$ \\
\hline Medium 2 = Clay & $\varepsilon_{r}=6.5, \sigma=0.001, \mu_{r}=1, \sigma_{*}=0$ \\
\hline
\end{tabular}

These properties remain constant throughout all calculated models. At first, the models consisted of only the depth information of the 414 Clay seam. Sequentially, additional random or stray clays were introduced into the models. Variation in the concentration of stray clay was then used to develop increasingly difficult data sets that will later be used to evaluate the performance of different auto-picking algorithms. To visually examine the numerical models created for any irregularities or discrepancies, ParaView [40] software was used. 
A 1D (A-scan) response in gprMax was created by positioning the antenna at the center of the model and recording the two-way travel-time (TWTT) response. Multiple AScans are then generated by moving the antenna from the start of the domain to the end. The collection of these A-scans over the model and their sequential plotting with a color intensity produce a corresponding 2D section (B-scan) of the model.

The different parameters considered in the numerical modeling here are:

1. Domain

Domain is the region where the antennas and realistic mediums are modeled in. For example, if the domain size is $2 \mathrm{~m} \times 1.5 \mathrm{~m} \times 0.002 \mathrm{~m}$ then all the source, receiver, clay seam, point reflectors and other possible components should exist within defined boundary.

\section{Time Window}

This is the total time required to generate one A-scan in a simulation. The time window can be calculated, as it forms the basis for recording/capturing the two way travelling time (i.e. the time it takes between the transmitter and receiver) of the model.

\section{Time Step}

Choosing the right spatial discretization for a model is dependent on the accuracy of the result and geometry of the target. A rule of thumb indicates that the spatial discretization should be at least ten times the wavelength of the signal [35]. 
However, for this research, the Yee cell size is $0.002 \mathrm{~m} * 0.002 \mathrm{~m} * 0.002 \mathrm{~m}$ used to capture all targets present in my model (stray clay exist in the model with are modelled as a blob). The model is a 2D TMz (Transverse Magnetic) model. The Yee cell for all the models considered in this research remains the same. Hence the time step also remains the same for all the models.

\section{Material}

The type of material for every medium in a numerical model must be specified. For this research, two mediums (salt and clay) are considered in the model and the properties of the material for these mediums are: Relative permittivity $\epsilon_{\mathrm{r}}$, Conductivity (Siemens/meter) $\sigma$, Relative permeability $\mu_{\mathrm{r}}$, Magnetic loss (Ohms/meter) $\sigma^{*}$. Table 3.2 shows their respective values.

\section{Waveform}

The type of waveform the source emits is also considered during modeling as the behavior of the waveform varies during reflection. This variation is caused by material properties in the domain. For this research, a point source (dipole) antenna with a $1000 \mathrm{MHz}$ frequency is used. The separation between the transmitter and receiver of the antenna is said to be $40 \mathrm{~cm}$.

\section{Model Geometry}


Different geometrical shapes must be considered while modeling. The shapes that were considered are cylindrical, thin bars, triangular patch in form of stray clay, non-uniform soil profile.

\section{Generation of A-scan}

In generating an A-scan, the antenna is usually placed at the center of the surface/roof and a single GPR trace is recorded.

\section{Generation of B-scan}

Series of A-scan are generated by the discretized movement of the GPR antenna through a model which takes every trace at each step. . B-scan, on the other hand, is the collection of multiple A-scan which displays a radargram of the earth modelwith a color intensity.

\section{Assumptions}

The assumptions made for the gprMax models are:

- The mediums used in this thesis are linear and isotropic.

- The GPR antenna is modelled as a point source using a Hertz dipole in gprMax.

- The material properties are fixed throughout the simulation.

- Salt is modelled as pure salt in gprMax. It is not lossy (zero conductivity) (table 2.1). 
- Clay is modelled as a lossy medium because generally the soil property exhibits some conductivity (table 2.1).

\subsection{Random generation of $\mathbf{4 1 4}$ clay seam profile}

The geophysicist's interpretation of the GPR data (ground truth) is one way of generating the soil profile for the simulator. Another method is to randomly generate clay profile by statistical methods to vary and change the structure of the soil profile into more complex data used to generate earth models. The random distribution of the clay profile leads to rough interfaces between different mediums. The characteristics of a given clay surface profile $y(x)$ can be determined using equations 3.1 and 3.2 to find the mean surface height $(\sim 1 \mathrm{~m})$ and standard deviation of the 414 clay seam.

$$
\begin{gathered}
\bar{y}=\frac{1}{N} \sum_{i=1}^{N} y_{i} \\
y_{S D}=\left[\frac{1}{N-1}\left(\sum_{i=1}^{N}\left(y_{i}\right)^{2}-N(\bar{y})^{2}\right)\right]^{1 / 2}
\end{gathered}
$$

Where,

$\mathrm{N}=$ Number of sample points

$\bar{y}=$ Mean surface height

$y_{S D}=$ Standard deviation

In order to randomly generate realistic soil profile, it is necessary to specify the standard deviation and surface correlation length of the clay profile (414 clay seam). A 
one-dimensional array of randomly generated numbers using Gaussian distribution representing the soil profile is produced with height points $y(x)$ with a mean of $1 \mathrm{~m}$.

The first parameter to describe the soil profile is to specify standard deviation or root mean square (rms) of the surface height $-h_{r m s}$.

The second parameter is to specify the correlation length $c l$, which is used in find the correlation function $C(y)$ (Eq. 3.3).

$$
C(y)=\exp \left(\frac{y^{2}}{c l^{2}}\right)
$$

The height function of the rough surface $R(y)$ is the convolution of the standard deviation and the correlation function (Eq. 3.4).

$$
R(y)=h_{r m s}^{2} \times C(y)
$$

In order to remove unwanted frequencies, the height function $R(y)$ is convolved with the power spectrum $p(y)$. Eq. 3.5 describes the power spectrum density (PSD) of a stationary Gaussian height distribution.

$$
p(y)=\frac{c l}{2 \sqrt{\pi}} \exp \left(-\frac{y^{2} c l^{2}}{4}\right)
$$

The result of the convolution above is then transformed into the time domain using the Inverse Fourier Transform (IFT). The real part of the result is the desired random generated soil profile. Detailed explanation of the theory behind random rough surface generator can be found in [34] [39].

The algorithm is written in MATLAB and the result is exported as a text file to gprMax to generate the numerical model. Fig. 3.4 shows a randomly generated soil profile with a marker indicating thinning salt back towards the roof. 


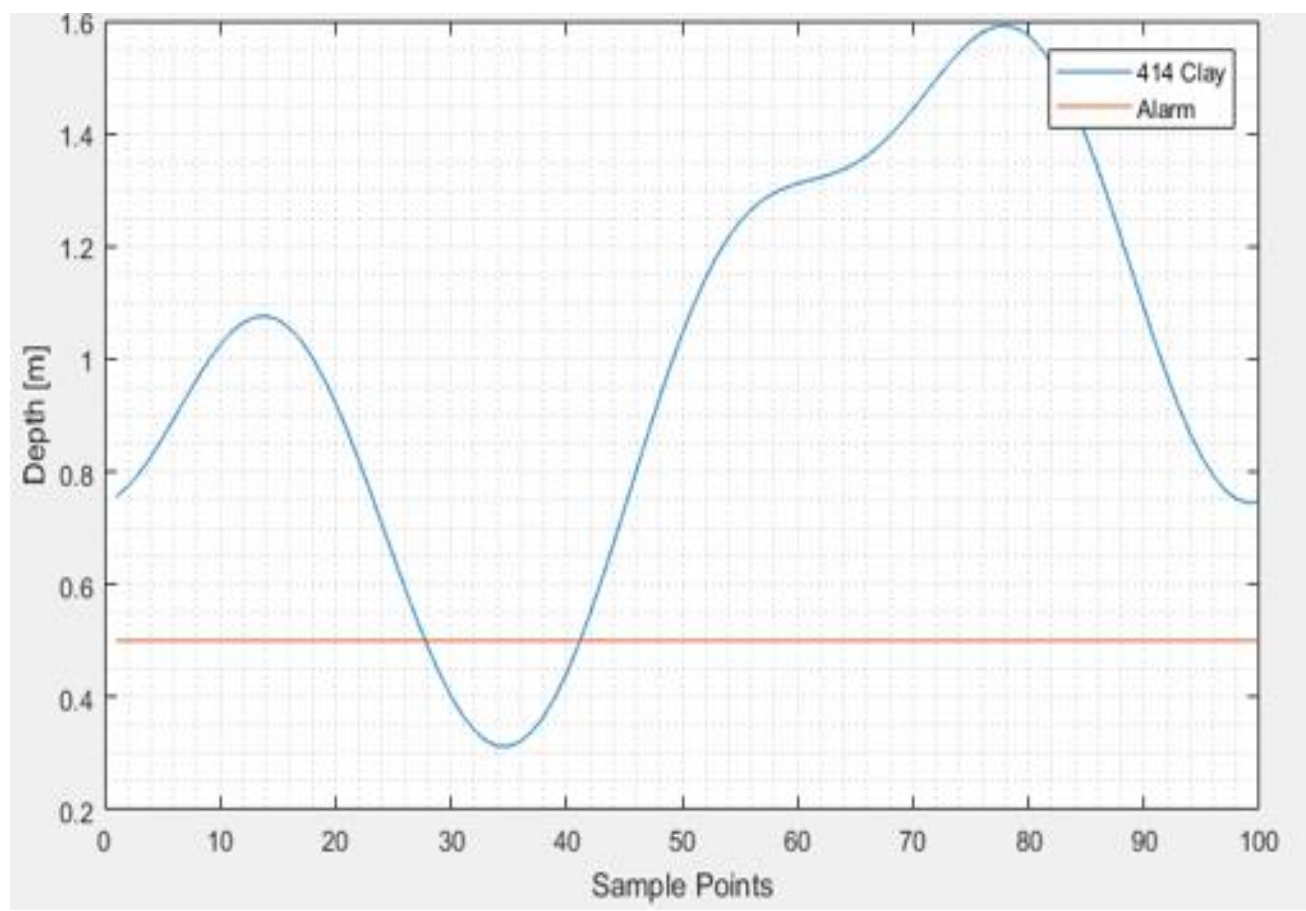

Fig. 3.4. Soil profile with a mean of $1 \mathrm{~m}$ and a deviation of $40 \mathrm{~cm}$.

\subsection{Stray clay}

Geological formation of potash sometimes creates bifurcation of clay (414 clay) in the earth. This bifurcation introduces stray clay scattered within the salt-back. These stray clays could be little pockets acting as point reflectors. They could also have visible structures or profiles like that of the actual 414 clay. This causes problem for any autopicking algorithm to find the right clay profile. The amplitude signal of these stray clay is affected by the depth, length and shape of the stray clay. In order to generate realistic earth models, two forms of stray clay are introduced into the model. The position of these stray clays is random. 


\section{Stray-Clay 1}

Stray-clay 1 is modelled in gprMax as clay pockets with a dimension of $0.02 \mathrm{~m} \times 0.01 \mathrm{~m} \times 0.002$. This introduces some level of noise into the synthetic data because in reality, the earth model is not ideal. In order to incorporate stray-clay1 into the model, the equation below is used.

$$
\% \text { stray. clay } 1=\frac{M * N}{R O I} * \frac{100}{1}
$$

Where:

$\mathrm{M}=$ Volume of stray-clay 1.

$\mathrm{N}=$ Number of stray-clay 1 .

$\mathrm{ROI}=$ Volume of region of interest in model domain.

The ROI is usually located within $20 \mathrm{~cm}$ to $100 \mathrm{~cm}$ of the salt-back thickness. Fig. 3.5 shows how stray-clay 1 is modelled within the ROI.

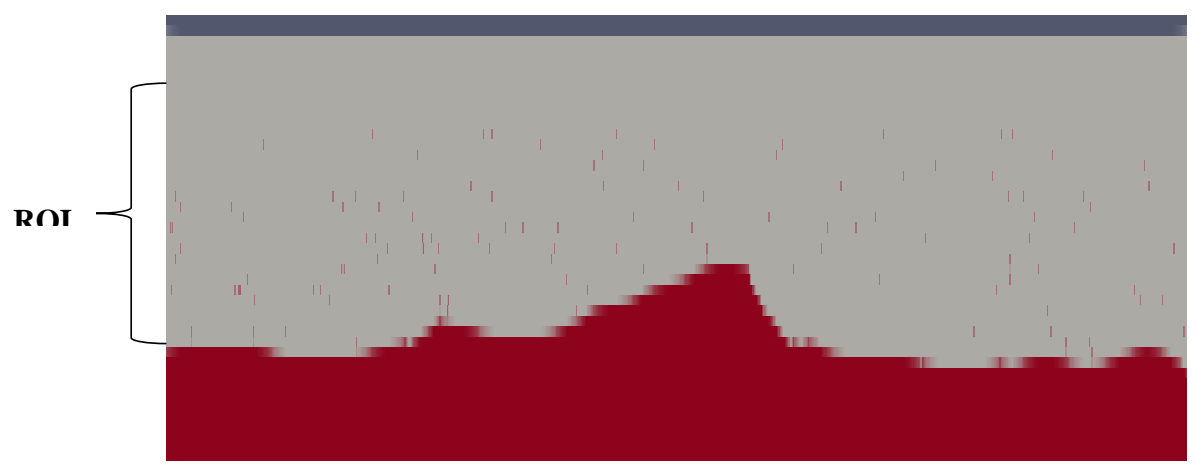

Fig. 3.5. Example of gprMax model with stray-clay 1 visualized with Paraview. 


\section{Stray-clay 2}

Stray-clay 2 is introduced into the model generator as distinct stray clay seen within the salt back. gprMax is used to create two triangular patches placed on each other. Fig. 3.6 shows the two triangular patches placed on each other with triangle one modelled as clay and triangle two modelled as salt. The apex angle for both triangles is the same but randomly generated. The apex of the triangle is varied from 170 degrees to 180 degrees and also in the inverted or negated form to generate an upright and inverted 'V-like' geometry. For every random apex angle, a resulting height is calculated to vary the arc of the 'V-like' stray clay. Stray-clay 2 is then generated into the model using the equation below.

$$
\% \text { stray. clay } 2=\frac{P * N}{R O I} * \frac{100}{1}
$$

Where:

$\mathrm{P}=$ Volume of triangle $1-$ volume of triangle 2

$\mathrm{N}=$ Number of stray-clay 2

ROI $=$ Volume of region of interest in model domain

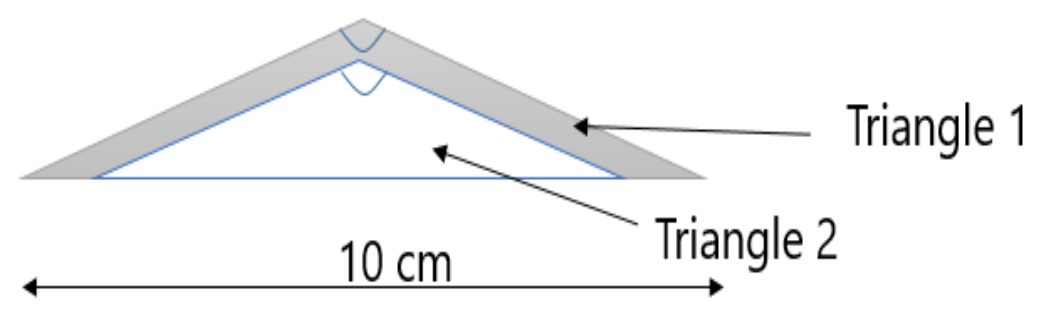

Fig. 3.6. Geometry of Stray-clay 2 . 


\subsection{Flow of data through software}

There exists a variety of software - many of which have multiple applications that are useful depending on the objective(s), relevance, and/or user preference. These software can be combined or used independently to generate and analyze numerical data for auto-picking algorithms (Fig. 3.7). For this research, the following software were used:

1. $\operatorname{gprMax}$

gprMax [35] is an open source software used for numerical modelling of GPR signals using the FDTD method to solve Maxwell's equations [35]. Although gprMax was intended for generating GPR data, it can also be used for other applications that require modeling of EM wave propagation. Python scripts are fed into gprMax to generate synthetic data.

2. Paraview

Paraview [40] which is a free software that uses the Visualization Toolkit (VTK) to display models created on gprMax.

\section{Amazon Cloud Computing}

The Amazon Cloud Computing [41] is a platform which allows users to own a virtual computer on the cloud depending on what kind of operation that 
needs to be performed. gprMax models are executed on the cloud which saves computational time required by the FDTD method.

\section{MATLAB}

MATLAB is a licensed software used to open and read files from gprMax. It makes it easier to edit and perform wiggle plots. The CRD algorithm is currently implemented on MATLAB.

\section{PYTHON}

Although python serves as one of the tools for gprMax, the python v2.7 has a script provided by Sensors \& Software to convert the output file of gprMax to an extension for use on a GPR machine. The extension is known as a .DT1 and .HD files.

\section{Ekko_Project}

Ekko_Project [23] is a licensed software provided by Sensors \& Software. It is used in processing and converting the .HD file to .SEGY file which can then be used in Matlab.

\section{IcePicker}

IcePicker [23] is a licensed software provided by Sensors \& Software. It is used to save or export the picks gotten from BackTrak to an excel format (.CSV) which can then be used on MATLAB to evaluate the performance of BackTrak. 


\section{BackTrak}

Designed by Sensors \& Software, BackTrak [13] is a software used for potash mine roof assessment. It is used for auto-picking and interpreting GPR data. A desktop application has recently been provided which enables users to run files generated with gprMax on BackTrak.

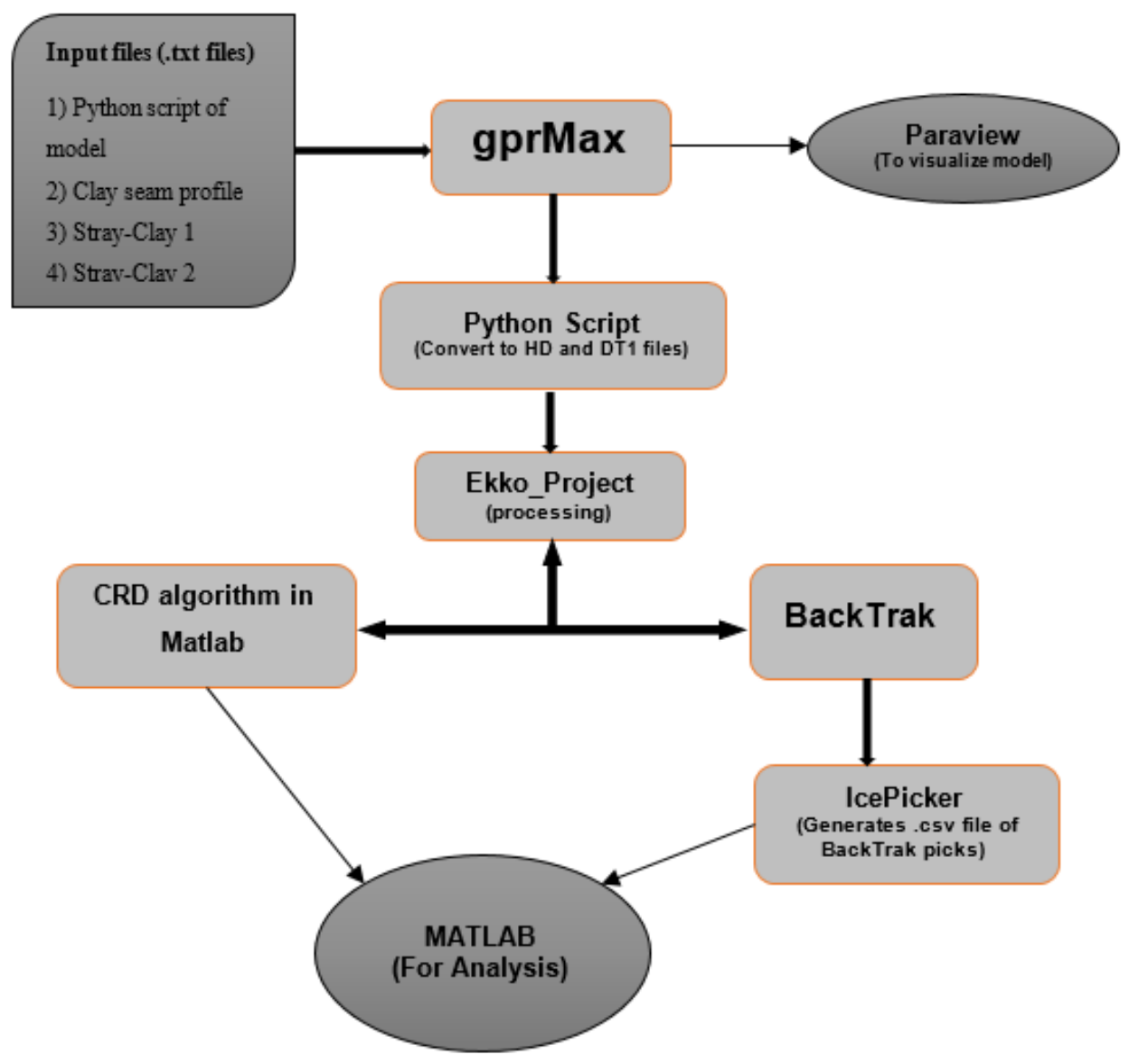

Fig. 3.7. Flow of data through software. 


\section{Chapter 4.}

\section{Results and Analysis}

The following section detail the results of validating the A-scan, core data measurement, and validating the B-scan with increasing complexity of anomaly ('stray clay'). The Sensors \& Software processing software - Ekko_Project - was used in processing the data. The steps taken in the processing of data are mentioned in section 3.2.1 of the actual data collection.

\subsection{A-scan validation}

To validate this method, a single GPR trace (A-scan) was chosen from the provided data set with a known salt-back thickness of $1.12 \mathrm{~m}$. A model using gprMax was used to create a synthetic A-Scan. A 2D model was then generated with a domain size of $2.0 \mathrm{~m} \mathrm{x}$ $1.5 \mathrm{~m} \times 0.002 \mathrm{~m}$, a center frequency of $1000 \mathrm{MHz}$, and the antenna positioned at the center of the domain. The upper part of the data represents salt while the lower part represents clay (Fig 4.1a). Stray clays with $0.02 \mathrm{~m}$ thickness and variable length are randomly placed within the salt-back to create a more realistic model. Fig 4.1b shows the A-scan plot comparison between the actual data and the synthetic data. It can be observed that the synthetic signal is very similar to the actual signal. 


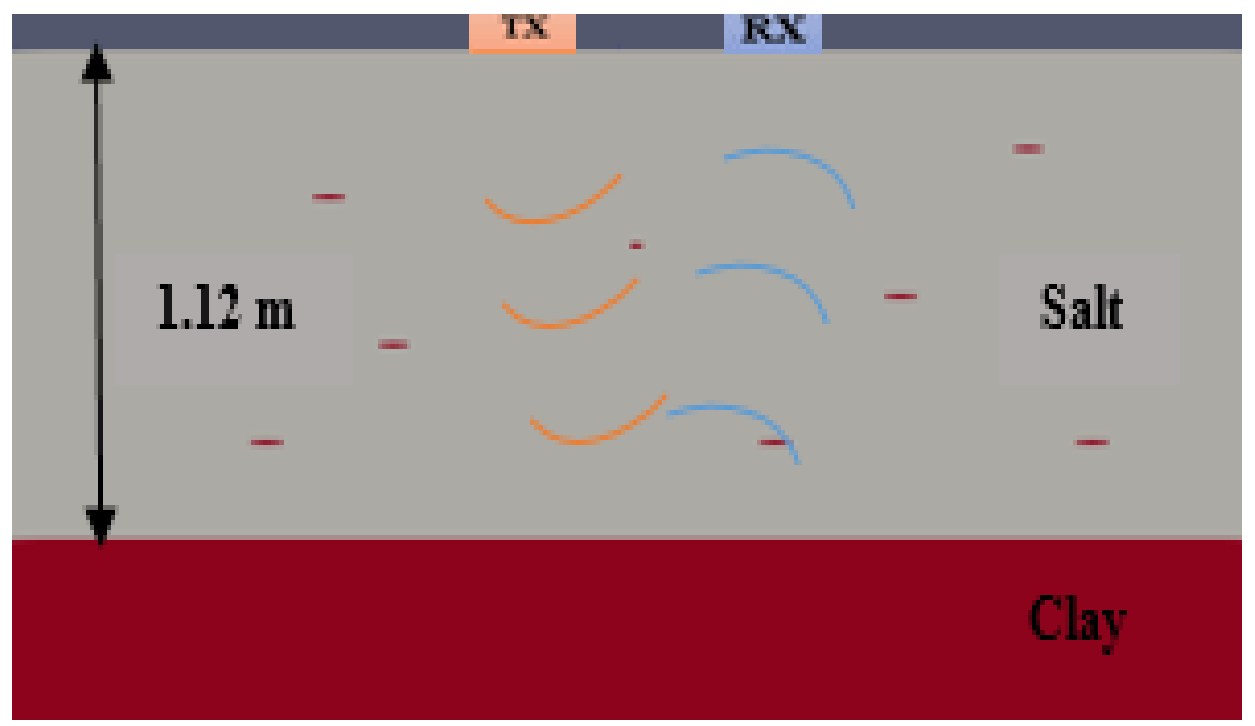

(a)

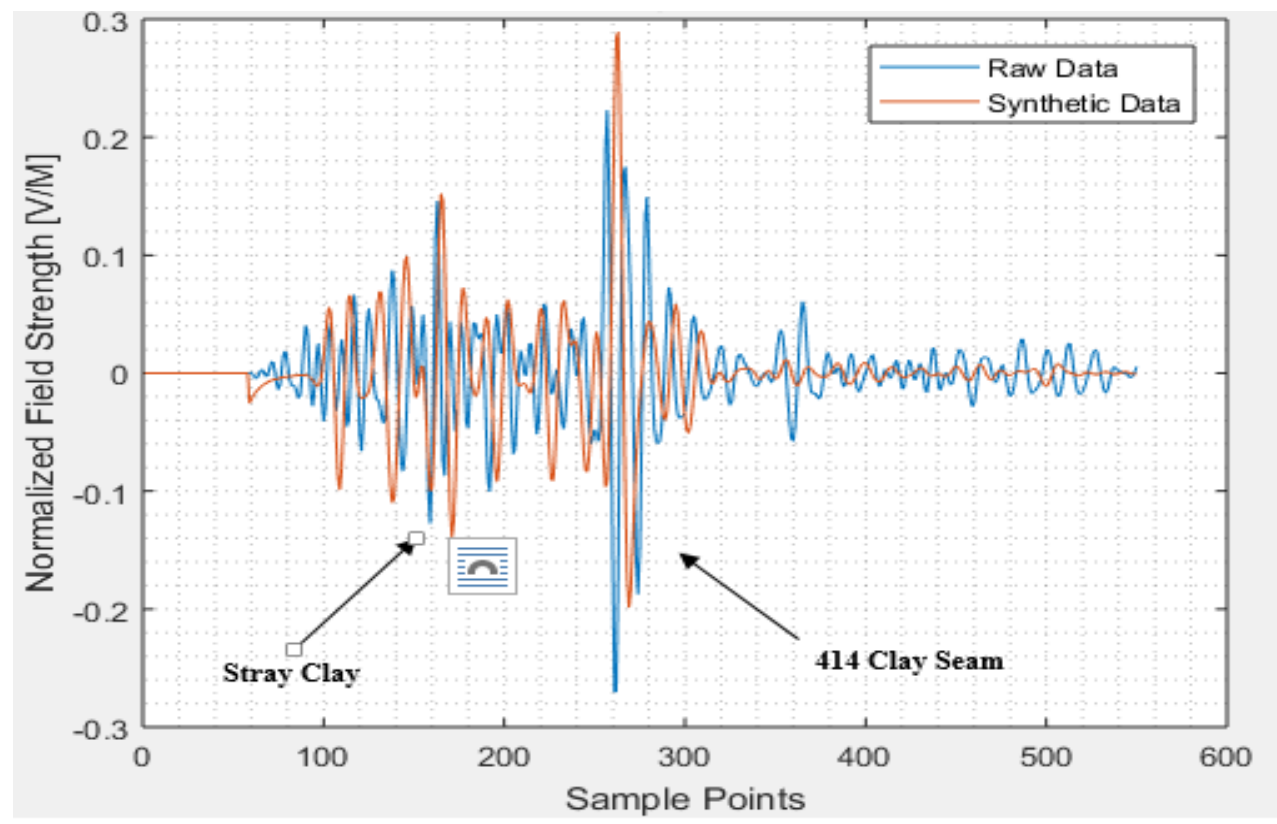

(b)

Fig. 4.1. (a) gprMax model. (b) A-Scan comparison between the actual and synthetic data of salt back thickness. It can be observed that the synthetic signal return is very similar to the actual signal. 


\subsection{Core data measurement}

In Fig. 3.2, core data were extracted from the GPR line at two different locations. Coring was done from the roof to the bottom of the 414 clay with a diameter of $5.5 \mathrm{~cm}$. This section describes how the physical measurement of this core data with the corresponding A-scans map to a numerically generated model of the actual measurement. Using the parameters presented in section 3.2.2, the antenna is placed at the center of the roof along the $\mathrm{x}$-axis and the measured stray clays are placed in the domain.

The overall aim of the experiment is to further validate that gprMax can be used to produce core data measurement and also compare it with the actual data. However, due to the limitation of coring the entire GPR line, historical and futuristic information (data) is unavailable to fully describe the geology of the cored area. This limits the validation of the core data with the numerical data to only identifying where the measured stray clays are, and where the measured 414 clay seam interface.

\subsubsection{Core 1}

The first coring occurred about $9.6 \mathrm{~cm}$ from the start of the GPR survey. The measured depth to the 414 clay seam is $115 \mathrm{~cm}$ with a measurement error of $\sim 4 \mathrm{~cm}$ in comparison with the geophysicist's interpretation. Two distinct stray clays are seen in the core data in Fig 4.2.

The first stray clay occurs at $81.4 \mathrm{~cm}$ depth from the roof with a $2 \mathrm{~cm}$ thickness. The second stray clay occurs $14.2 \mathrm{~cm}$ after the first stray clay. The resulting A-scan shows the reflections of the stray clays and the 414 clay seam of the synthetic data when compared 
to the actual data. Multiple reflections are seen after the interface of the second stray clay as a result of the mixing zone (mixture of salt and clay) present in the model.
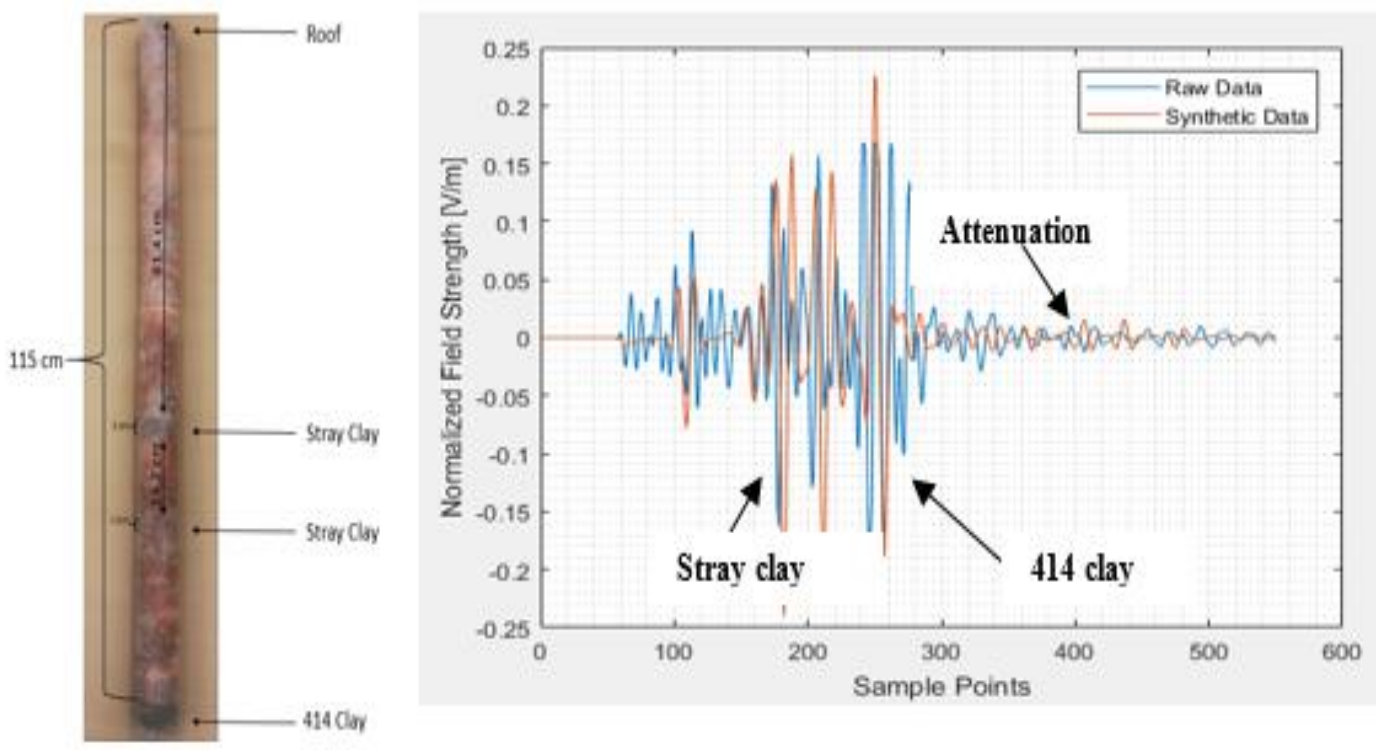

Fig. 4.2. Core-1 data with the corresponding A-scan.

\subsubsection{Core 2}

The measured depth of the second core data from the roof to 414 clay seam is $99.3 \mathrm{~cm}$ with a measurement error of $\sim 2 \mathrm{~cm}$ in comparison with the geophysicist's interpretation. Two stray clays are observed in the core data in Fig. 4.3. The first stray clay has a depth of $39 \mathrm{~cm}$ from the roof with a $2 \mathrm{~cm}$ thickness. The second stray clay occurs $29 \mathrm{~cm}$ after the first stray clay with a thickness of $1.8 \mathrm{~cm}$. 
The resulting A-scan shows the reflections of the stray clays and the 414 clay seam of the synthetic data when compared to the actual data. Noise is seen at the initial part of the signal just before the stray clay. This noise is as a result of little clay pockets in the earth geology that are not visible in the core data presented.
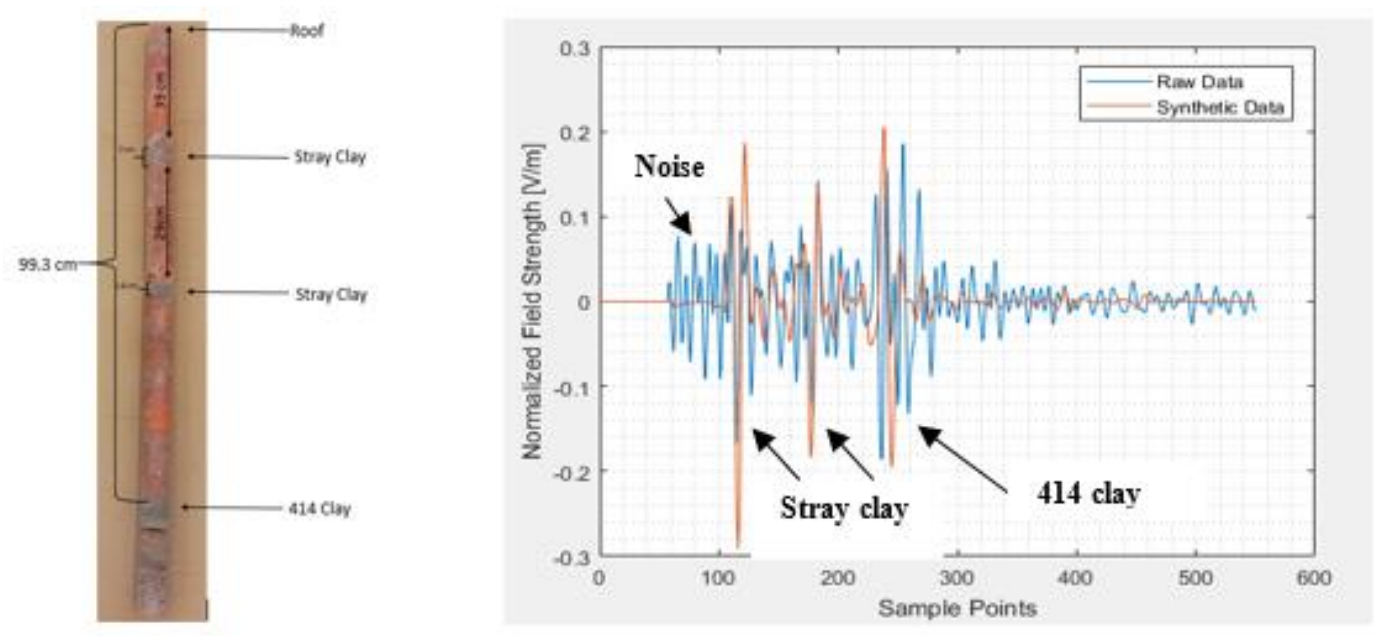

Fig. 4.3. Core-2 data with the corresponding A-scan.

\subsection{B-scan validation}

In validating the synthetic data set generated from the geophysicist's picks, the CRD algorithm and Bactrak is applied to the data set and compared with the geophysicist's picks. Fig. 4.4 shows the results of auto-picking applied to actual GPR data using both the BackTrak and the CRD algorithms. Both algorithms result in an accurate estimate of the 414 clay seam as picked by the geophysicist. This result supports the idea that autopicking algorithms can produce results that are similar to those of the geophysicist, and that both the BackTrak and CRD algorithms have comparable results. 


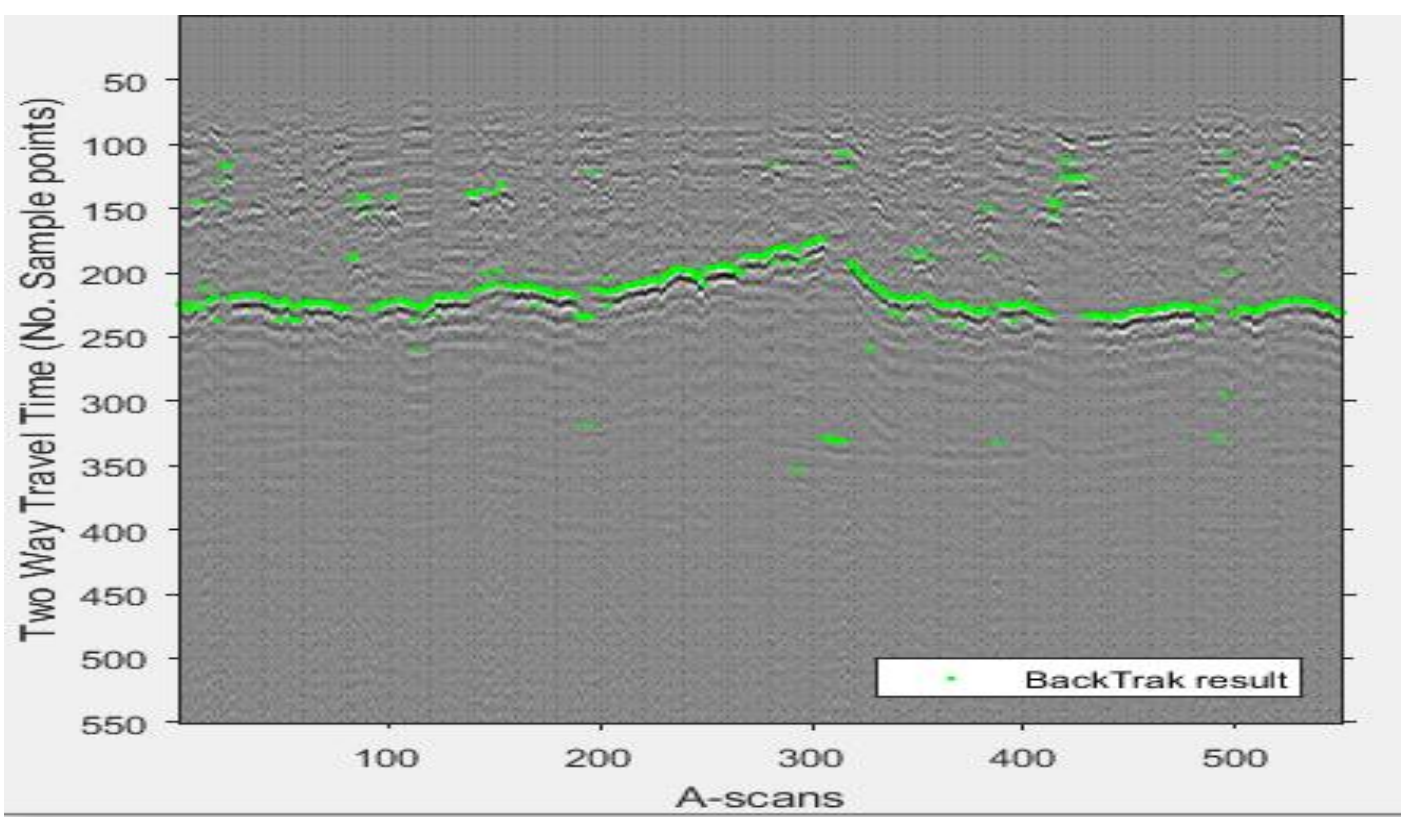

(a)

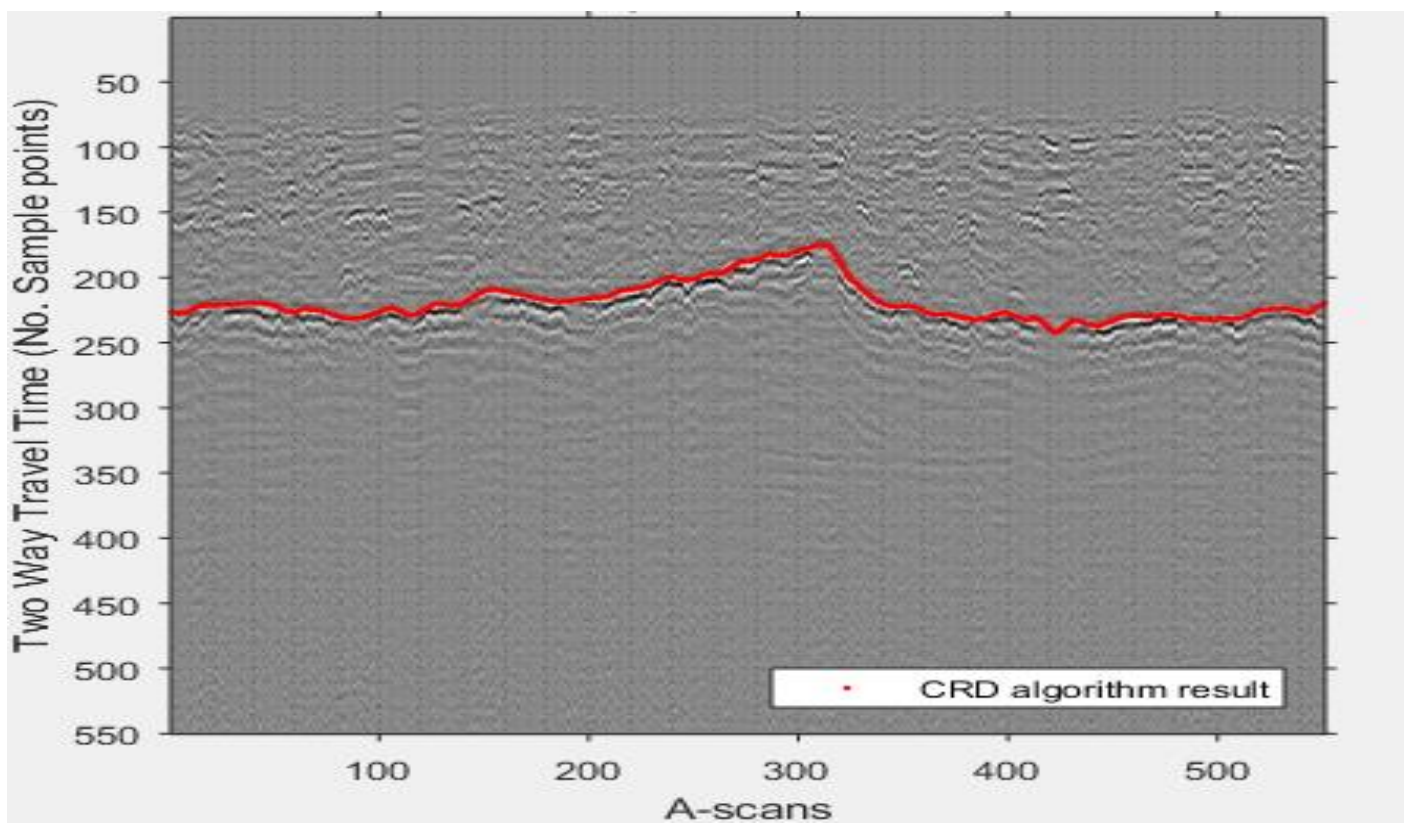

(b)

Fig. 4.4. Results of auto-picking techniques applied to the actual data. (a)

BackTrak picks imported into Matlab. (b) CRD algorithm plotted in Matlab. 
Four (4) experiments were considered with a progressive increase in the volume of stray-clay. Each model was built to replicate a sample data set of 540 GPR traces (Ascans). For these models, the depth of the 414 clay is known and modeled from the geophysicist interpretation. Therefore, the 'base' model consisted of only the 414 clay seam and for each sequential model, the percentage and length of 'stray-clay' was changed to create increasingly more complex model. The four experiments considered in this thesis are described in detail below. These models were built with the base parameters provided previously in Table 3.2.

\subsubsection{Experiment 1}

For this base model, the volume of stray-clay present is $0 \%$. Only the actual depth (i.e. geophysicist interpretation of salt back thickness) is presented. The resulting B-scan (Fig. 4.5) displays a distinct radargram of the corresponding model without any noise signals. 


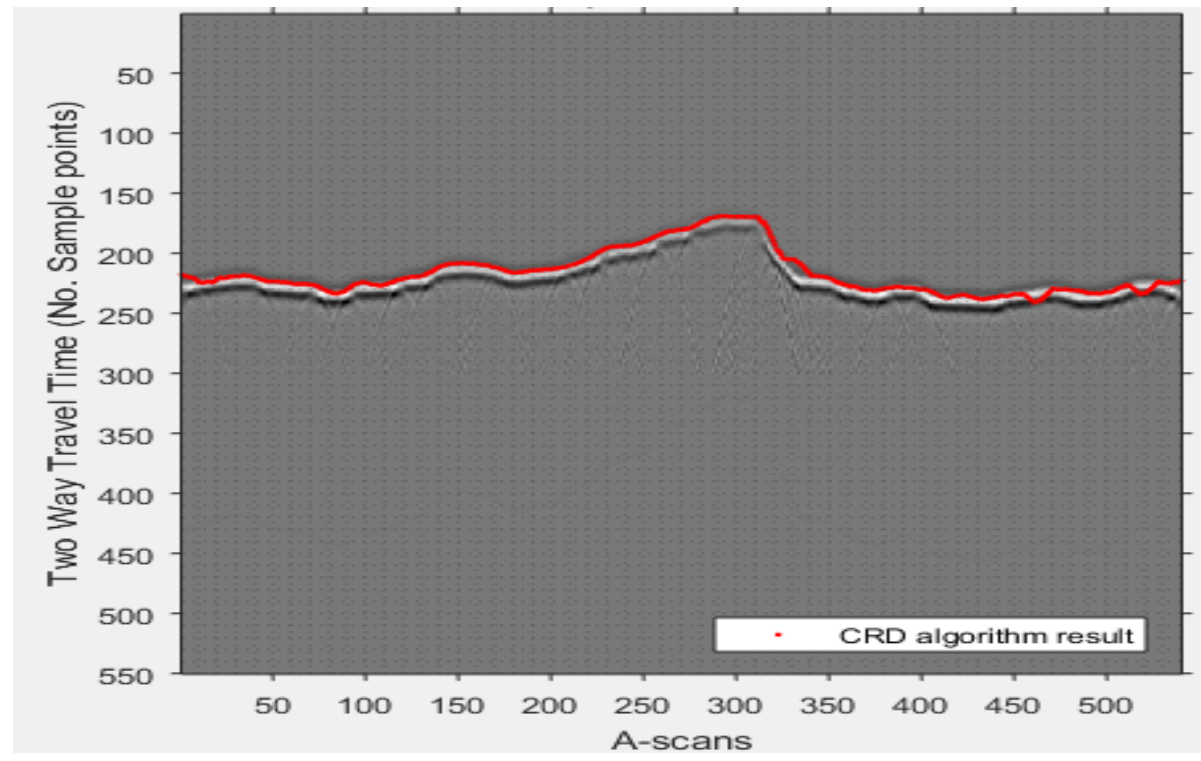

Fig. 4.5. B-scan of experiment 1 with CRD algorithm applied.

\subsubsection{Experiment 2}

The model in experiment 2 is an update to the previous model as it incorporates interspersed clay (stray-clay 1) randomly distributed within the salt-back. The dimension of the clay pockets is $0.02 \mathrm{~m} \times 0.01 \mathrm{~m} \times 0.002 \mathrm{~m}$ with a volume of $1.85 \%$ present in the model. A representation of this earth model used in this experiment is seen in the Fig. 4.6 and can be compared to the actual radargram (Fig. 4.4b). 


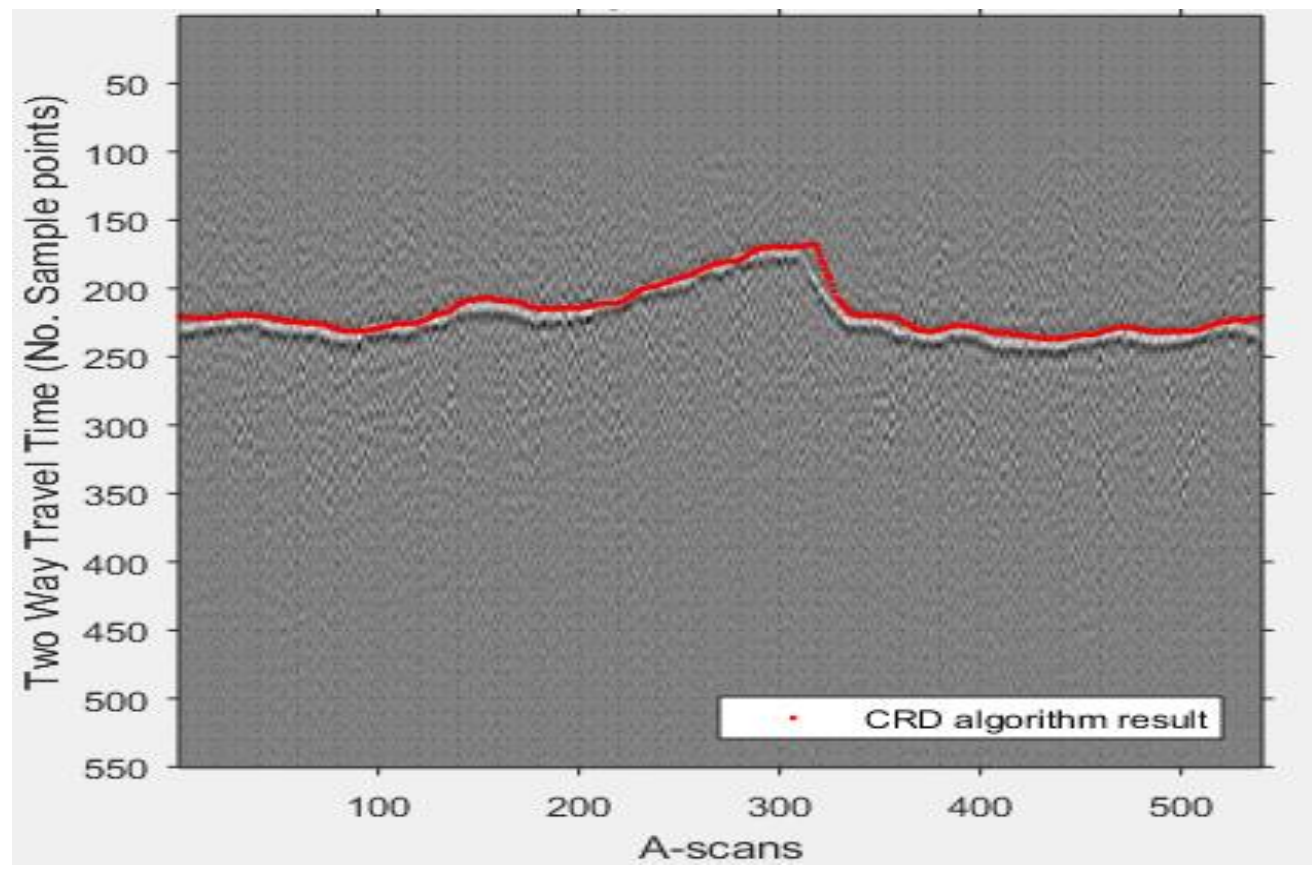

Fig. 4.6. B-scan of experiment 2 with CRD algorithm applied.

\subsubsection{Experiment 3}

Stray-clay 2 is introduced in experiment 3 and augments the incorporation of stray clay 1 from the previous experiment. This clay is modeled as a triangular patch with a primary angle varying between $175^{\circ}$ to $185^{\circ}$ to form the structure of the stray clay. The base length of the stray clay is $1 \mathrm{~m}$ with a thickness of $0.02 \mathrm{~m}$. The total volume of stray clay is $4.94 \%$ with stray-clay 2 component representing 3.09\%. The radargram (Fig. 4.7) shows the GPR with stray-clay 2 modeled. 


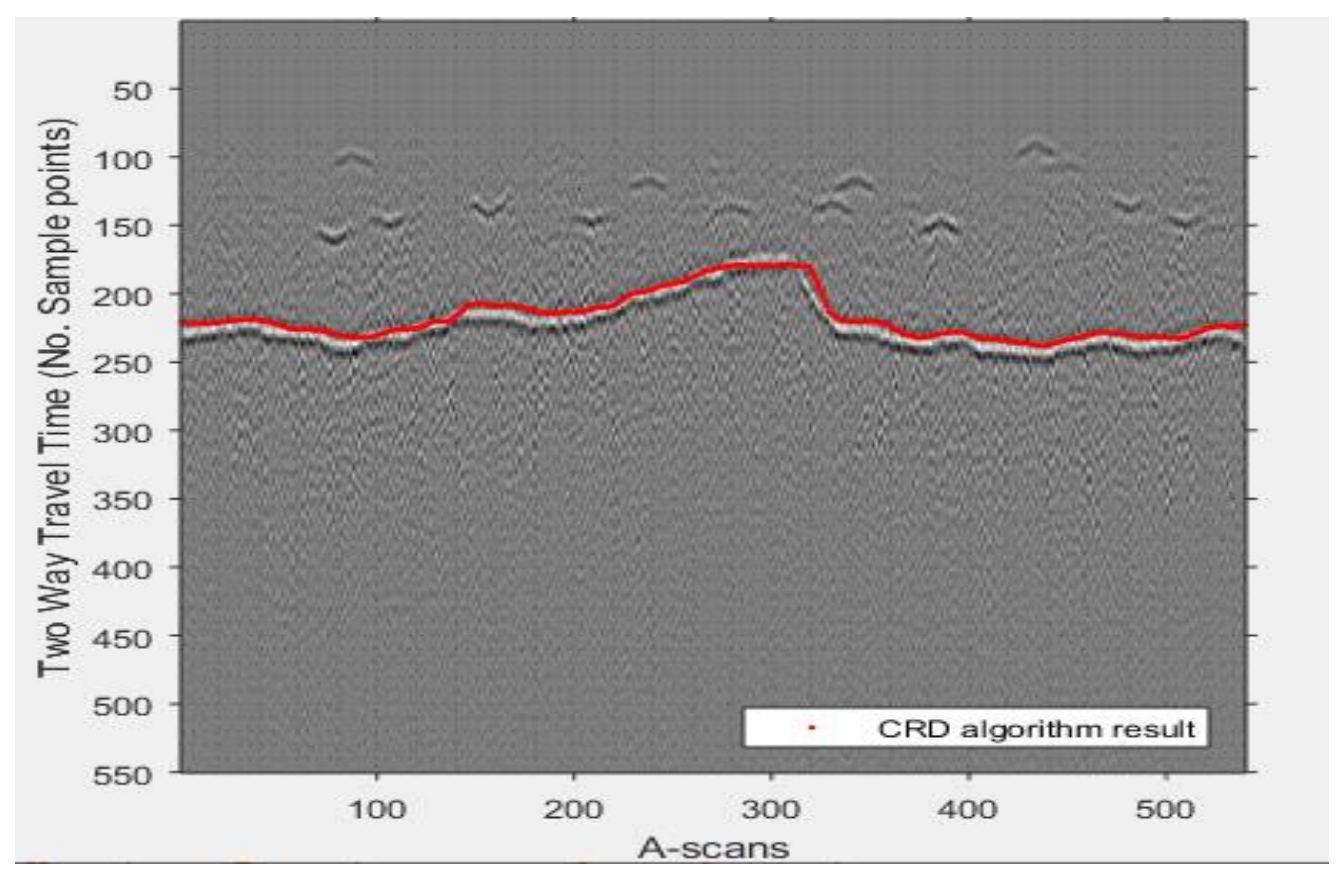

Fig. 4.7. B-scan of experiment 3 with CRD algorithm applied.

\subsubsection{Experiment 4}

The volume of stray-clay 2 in experiment 4 is further increased with the addition of rectangular patches of clay to the model. The dimension of the rectangular patch is $0.5 \mathrm{~m}$ x $0.02 \mathrm{~m} \times 0.002 \mathrm{~m}$. The total volume of stray clay present in the model becomes $8.62 \%$. Fig. 4.8 shows the resulting B-scan for this experiment. The CRD algorithm shows a poor performance as the volume of stray clay increases. 


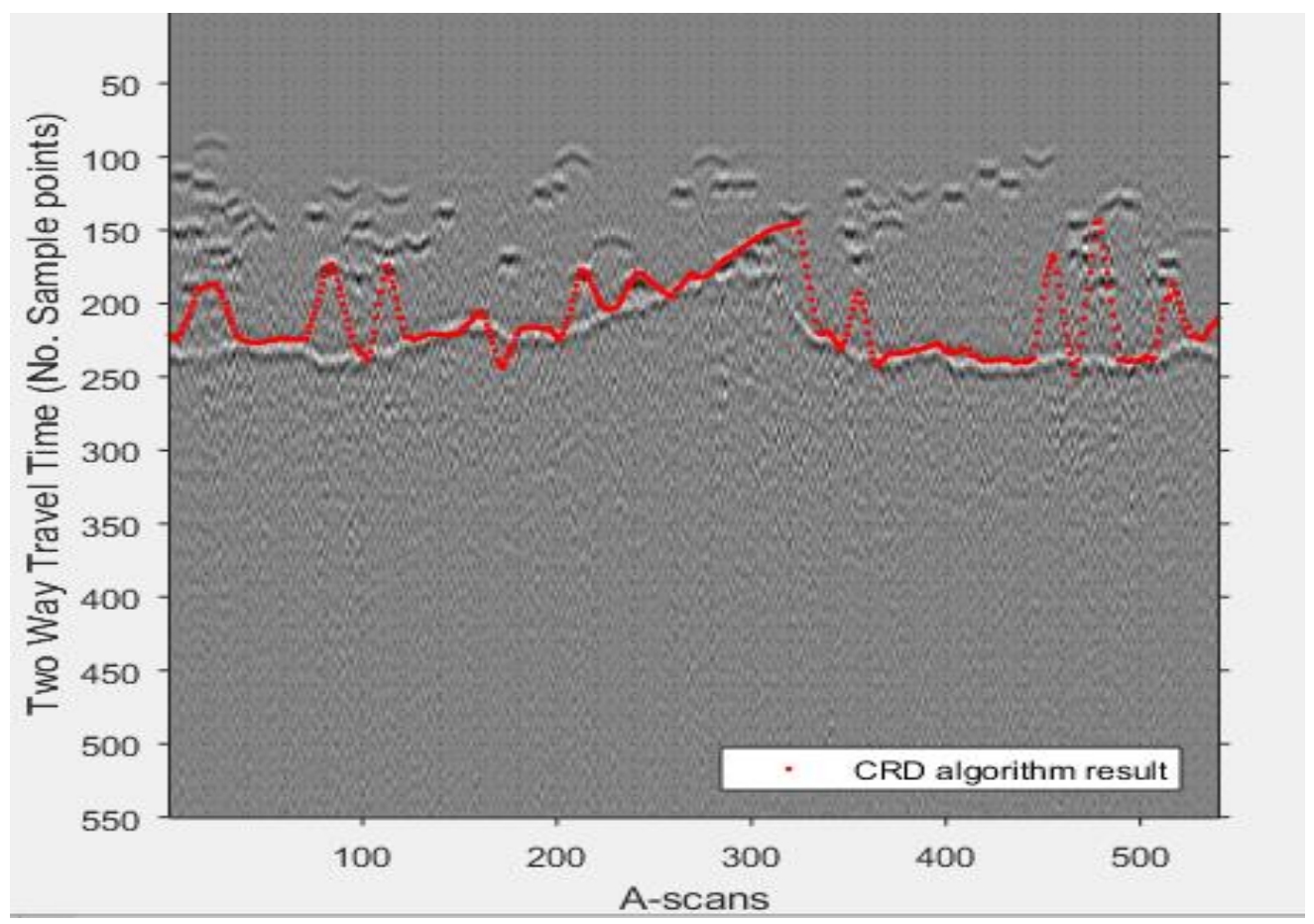

Fig. 4.8. $\quad$ B-scan of experiment 4 with CRD algorithm applied.

\subsection{Simulation analysis}

A summary of the experimental procedure as well as details of the experimental conditions and the effectiveness of the auto-picking algorithm are presented in table 4.1. The auto-picking results are similar for experiments 1 to 3 but different for experiment 4 where the CRD algorithm performs poorly. The percentage standard deviation (SD) is used to analyze how much deviation the picks of the CRD algorithm is from the ground truth (geophysicist's interpretation). 
Table 4.1. Overall simulation process

\begin{tabular}{|l|l|l|l|l|l|}
\hline Properties & Exp. 1 & Exp. 2 & Exp. 3 & Exp. 4 & $\begin{array}{l}\text { Actual } \\
\text { data }\end{array}$ \\
\hline $\begin{array}{l}\text { \% stray- } \\
\text { clay 1 }\end{array}$ & 0 & 1.85 & 1.85 & 1.85 & N/A \\
\hline $\begin{array}{l}\text { \% stray- } \\
\text { clay 2 }\end{array}$ & 0 & 0 & 3.09 & 6.77 & N/A \\
\hline $\begin{array}{l}\text { Total \% of } \\
\text { stray-clay }\end{array}$ & 0 & 1.85 & 4.94 & 8.62 & N/A \\
\hline $\begin{array}{l}\text { No. of } \\
\text { traces }\end{array}$ & 540 & 540 & 540 & 540 & 540 \\
\hline $\begin{array}{l}\text { CRD (\% } \\
\text { accuracy) }\end{array}$ & 97.3329 & 97.3151 & 96.9387 & 86.9262 & 98.7339 \\
\hline $\begin{array}{l}\text { CRD (\% } \\
\text { SD) }\end{array}$ & 2.6947 & 2.839 & 4.2674 & 19.5685 & 1.7161 \\
\hline
\end{tabular}

It is important to understand computational implications of this simulation system.

Table 4.2 presents data on the amount of time each simulation run requires. The simulation time window represents the depth information recorded from a single GPR trace. As the time window increases, the EM wave propagated further into the ground from the antenna and the GPR signal is recorded. The execution time between using Amazon Cloud Computing [11] and a typical 1-GPU (Graphics Processing Unit) processor computer platform to execute this simulation is also compared and shown in table 4.2. The table shows scale factor improvement in using the Amazon Cloud over a typical personal computer. It is observed that processing times are normally improved by a factor of about 10. 
Table 4.2. Simulation time of an ordinary PC without GPU vs. a virtual PC with 1GPU for an A-Scan

\begin{tabular}{|c|c|c|c|}
\hline $\begin{array}{c}\text { Simulation Time } \\
\text { Window [ns] }\end{array}$ & $\begin{array}{c}\text { Regular PC } \\
\text { (without GPU) } \\
\text { [secs] }\end{array}$ & $\begin{array}{c}\text { Amazon Cloud } \\
\text { (1-GPU [secs] }\end{array}$ & $\begin{array}{c}\text { Time Factor } \\
\text { Improvement }\end{array}$ \\
\hline 10 & 37.42 & 4.14 & 9.04 \\
\hline 20 & 70.46 & 8.92 & 7.90 \\
\hline 30 & 103.08 & 8.98 & 11.48 \\
\hline 40 & 135.83 & 12.03 & 11.29 \\
\hline 50 & 141.89 & 13.66 & 10.39 \\
\hline
\end{tabular}

A typical mine data consists of over 2,000 traces, which can be translated to over 100 meters distance. The result from table 4.2 shows that the time factor improvement for an A-scan shows a huge significance when applied to a large number of GPR traces (e.g. running over 10,000 GPR traces on the cloud can save over 27 days of computational time). 


\section{Chapter 5.}

\section{Conclusion and Future Work}

\subsection{Discussion and Conclusion}

In this thesis, a set of actual GPR data from a mine was used to evaluate how representative an earth model and the GPR simulator (gprMax) can be used. The A-scan comparison of the actual and synthetic data demonstrates that for both data sets, the reflections from the 414 clay seam will have a significant peak at different depths depending on the salt-back thickness (Fig. 4.1). Further, it is observed from the simulation (Fig. 4.1) that additional 'stray clay' can produce confounding reflections for the autopicking algorithm as it suppresses the EM reflected response from the 414 clay seam.

The B-scan generated in this thesis shows and validates that this simulation system is representative of what the boring machine operator sees during mining. The experimental results presented in this work demonstrate the value of using a simulation system to generate GPR data as a test bed for auto-picking algorithms. The CRD algorithm is used in validating the accuracy of the earth models generated in this work. A point by point subtraction of the position of the auto-picking algorithm's picks with those of the geophysicist's picks was done and averaged to assess the effectiveness of the algorithm. The results from Table 4.1 show that the auto-picking algorithm validated the geophysicist's picks with an error of $<3 \%$. However, this thesis shows that as the volume of stray clay increases, the accuracy of the algorithm decreases. There is an increase in 
the percentage deviation (SD) of the algorithm's pick from the 414 clay seam from $2.69 \%$ to $19.57 \%$ through Experiments $1-4$ (sections 4.3 .1 - 4.3.4). Fig. 4.8 shows the progression in the volume of stray clay and performance of the CRD algorithm. The decrease in the accuracy of the auto-picking pick is as a result of the stray clays injected into the system. The reflection of stray clay is affected by the length and depth of the stray clay which creates difficulties for auto-picking algorithms. In addition, by using cloud computing we demonstrate that the time taken to generate these models is reasonable and practical. Also, the results in Table 4.2 indicate that on average, there is a time improvement factor of 10 in data generation time.

It is expected that the earth model simulator will serve as a test bed for new and improved auto-picking algorithms. The ultimate goal is to improve the safety of potash mining, and through the development of good simulators, good auto-picking algorithms for potash mine application can be developed.

\subsection{Contribution}

In this thesis, I have successfully developed a software tool to generate various earth models using the geophysicist's interpretation or a random earth profile generator as the input file into gprMax simulator. Also, I have defined a method of introducing stray clay into the model as another input file for the simulator. These stray clays are called stray-clay 1 (point clay reflectors acting as noise) and stray-clay 2 (upright and inverted V-shape stray clay) respectively. Thus, the user can specify the characteristics of the soil 
profile and the percentage of stray clay required for a particular model. My model generator uses the parameters described in table 3.1, calculates the number of traces (Ascan) required and through a step size, gprMax simulates the EM wave into my model to generate the synthetic GPR data.

My overall thesis demonstrates that we can successfully generate synthetic GPR signals that is similar to the actual data present in the mine. Thus, leading us to confidently generate test bed data with known ground truth for auto-picking algorithms applicable to potash mine.

\subsection{Limitations and future work}

gprMax software has proven to be a reliable tool for generating GPR data that can be compared to potash mine data. However, quantitative measurement and simulation of core data does not completely represent the actual GPR signal (Figs. 4.2 and 4.3). Due to the propagation of EM wave from the GPR device, core data samples might be insufficient to accurately measure and model the earth as previous and future information of the earth geology is unknown.

Another limitation is that in-situ measurement of material properties (dielectric constant and electrical conductivity) of salt and clay is difficult to obtain because when the earth is cored, the material properties change due to exposure to air (moist environment). Therefore, the material properties used in generating the synthetic data were based on literature review and assumptions made. 
A 2-D analysis of the real world is also a limitation in this work. The data collection process in 2-D is not sufficient enough to describe/determine the return signal from the GPR device. The diffraction of the EM wave into the roof makes it possible to detect stray clays that exists within a 3-D world.

Future work can be done on proper measurement of stray clay positions within the earth model. The use of image processing techniques or some form of signal processing techniques to give an estimate of the discrete position of stray clays, due to the limitations that exist from coring the entire roof.

The computational time required for generating a synthetic signal depends upon the Yee cell and the domain size. Future work can also be done on adequate sampling of the Yee cell used in the model by applying the rule of thumb described in gprMax to save computational time while maintaining the accuracy of the result.

Several anomalies that can cause ground fall during mining exist within the saltback such as stray clays, wash-outs, mixing zones for clay, salt and potash. This thesis focuses mainly on modeling stray clays within the salt-back. Therefore, it is recommended that other important geological features (i.e. wash-outs, mixing zones) will be addressed in future earth models, as these features can also confound auto-picking algorithms. 


\section{References}

1. Anne Fuzesy "Potash in Saskatchewan" Saskatchewan Energy Mines, 181 (1982), p. 2.

2. Funk, C.W., R. Brehm, A.F.C. Errington, and K. Backstrom, 2016, “An Apparatus for Collecting Upward-looking GPR Surveys in Potash Mines”, 16th International Conference of Ground Penetrating Radar, Hong Kong, China.

3. P. R. Jones, F. F. Prugger, "Underground mining in Saskatchewan potash", Mining Engineering, vol. 34, pp. 1677-1683, 1982.

4. A. M. Coode, M. Rizkalla and Y. Potvin, "Towards a Better Understanding of the Deflection of Limestone Beds above Evaporites," 1996.

5. E. M. D. Souza and A. M. Coode, "Control of sodium-rich brine inflows at Central Canada Potash - Part 2: Rock mechanics, instrumentation and results," CIM Mining Technology, pp. 87 - 92, 1990.

6. D. Stead, E. Eberhardt and Z. Szczepanik, "Brittle rock fracture and progressive damage in potash," in Proceedings of the 8th World Salt Symposium, the Hague, Oxford, 2000.

7. C. Boys, "The Geology of Potash Deposits at PCS Cory Mine, Saskatchewan" (Master of Science Thesis), Saskatoon, Saskatchewan: University of Saskatchewan, 1990.

8. Daniels, D. J., 'Ground penetrating radar', IEEE, 2nd edition, The University of Michigan, 2004. 
9. D. Daniels, "Surface-penetrating radar", 2nd ed. London: Institution of Electrical Engineers, 2004, pp. Chp 8 - 14.

10. Jol, H.M., "Ground Penetrating Radar Theory and Applications”, 2009, London: Elsevier Science. 524.

11. Do, J., "Report: Ground Penetrating Radar", 2003, Villanova University: Pennsylvania. p. 5.

12. Brooks, J.W., "The Detection of Buried Non-Metallic Anti-Personnel Land Mines", in Department of Electrical and Computer Engineering. 2000, University of Alabama: Huntsville. p. 127.

13. Belli, K.M., "Ground Penetrating Radar Bridge Deck Investigations Using Computational Modeling", in College of Engineering, Department of Mechanical and Industrial Engineering. 2008, Northeastern University: Boston. p. 235.

14. Abujarad, F., "Ground Penetrating Radar Signal Processing For Landmine Detection", in Institute of Electronics, Signal Processing and Communications Engineering (IESK). 2007, University of Magdeburg: Magdeburg, Germany. p. 124.

15. Huston, B., et al., "Non-destructive Testing of Reinforced Concrete Bridges using Radar Imaging Techniques”. 2002, Department of Mechanical Engineering, College of Engineering and Mathematics, University of Vermont: Burlington. p. 182.

16. Jeng Y., Lin C., Li Y., Chen C. and Huang, H., “Application of multi-resolution analysis in removing ground penetrating radar noise," in CSPG CSEG CWLS Convention, (Calgary, Canada), pp. 416-419, 2009. 
17. O. Yilmaz, "Seismic Data Processing", Society of Exploration Geophysicists, Tulsa, OK.

18. E. Fisher, M. George and P. Annan, "Acqusition and processing of wide-aperture ground-penetrating radar data," Geophysics, vol. 3, no. 495504, p. 57.

19. C. A. Balanis, "Advanced Engineering Electromagnetics", chapter Electrical properties of matter, Wave equation and its solutions, pages 42-126. John Wiley \& Sons, 1989.

20. Daniels, D. J., editor (2004). "Ground Penetration Radar", 2nd Edition. The Institution of Electrical Engineers, London.

21. S. K. Yee, "Numerical solution of initial boundary value problems involving maxwell's equations in isotropic media”. IEEE Transaction on Antennas and Propagation, 1996.

22. A. Taflove, and S. C. Hagness, "Computational Electrodynamics: the finitedifference time-domain method", 2nd edition. Artech House,Inc, 2000.

23. S. \&. Software. [Online]. Available: https://www.sensoft.ca/.

24. T. K. Briggs, ”Auto-picking Algorithm Development For Hidden Clay Seams In Potash Mines”, M.Sc thesis, University of Regina, Regina, SK, 2018.

25. Y. Vaezi and M. Van der Baan, "Comparison of the STA/LTA and power spectral density methods for microseismic event detection", Geophysical Journal International, vol. 203, no. 3, pp. 1896-1908, 2015.

26. B. Sharma, A. Kumar and V. Murthy, "Evaluation of seismic events detection algorithms", Journal of the Geological Society of India, vol. 75, no. 3, pp. 533$538,2010$. 
27. B. B. Bannatyne, "Devonian potash deposits in Manitoba" Manitoba Department of Energy and Mines, Mineral Resources Division, Open File Report OF83-3, 27 p, 1983.

28. G. R. Guillet and W. Martin (ed.), A. Fuzesy, "Potash in western Canada; in The Geology of Industrial Minerals in Canada", Canadian Institute of Mining and Metallurgy, Special Volume 29, p. 188-194, 1984.

29. C. Funk, J. Appleyard, J. Braun and J. Derkach, "How Geoscientists are Making Potash Mines Safer," CSEG RECORDER, vol. 42, no. 04, pp. 12-17, June 2017.

30. J. W. Shirley, "An early experimental determination of Snell's law," Am. J. Phys. 19, 507-508 (1951).

31. A. Annan, J. Davis and D. Gendzwill, "Radar sounding in potash mines, Saskatchewan, Canada”, GEOPHYSICS, vol. 53, no. 12, pp. 1556-1564, 1988.

32. P. Anna, "Ground Penetrating Radar Principles Application and Procedure", 2003

33. G. G. Clemeña, Short-Pulse Radar Methods." In: Handbook on Nondestructive Testing of Concrete, Malhotra, V.M and Carino, N.J., ed., 2nd Ed., ASTM \& CRC, 2004.

34. N. Garcia, E. Stoll, "Monte Carlo Calculation of Electromagnetic-Wave Scattering from Random Rough Surfaces", Physical Review Letters, Volume 52, Issue 20, pp. 1798-1801 (1984).

35. Giannopoulos, A. (2005) GprMax2D/3D User Guide Version 2.0. 69.

36. S.Valle, L. Zanzi, and G. Lenzi, "2D and 3D focusing of ground penetrating radar data for NDT", Proc. SPIE 4084, Eight international Conference on Ground Penetrating Radar, (27 April 2000). 
37. A. Neal, "Ground-penetrating radar and its use in sedimentology: principles, problems and progress". Earth science reviews 66: 261-330, 2004.

38. A. P. Annan, DK Butler (ed), "Ground-penetrating radar. In Near surface geophysics", Society of exploration geophysicists: Tulsa, Investigations in Geophysics 13; 357-438, 2005.

39. Tsang, L., Kong, J. A., and Ding, K, "Scattering of electromagnetic waves: Theories and applications", chapter Characteristics of discrete scatterers and rough surfaces, pages 167-197. John Wiley and Sons, Inc., 2000.

40. Ahrens, James, Geveci, Berk, Law, Charles, ParaView: An End-User Tool for Large Data Visualization, Visualization Handbook, Elsevier, 2005, ISBN-13: 9780123875822.

41. Amazon Web Services. [Online]. Available: https://ec2.amazonaws.com/ 


\section{Appendix A.}

\section{Code Scripts}

\section{Python Script for gprMax}

\#title: B-scan of a Salt Back(Roof to 414 Clay) with Normal thickness of 1m (Stray-Clay) domain: 2.01 .500 .002

\#dx_dy_dz: 0.0020 .0020 .002

\#time_window: 30e-9

\#material: 2.7010 salt

\#material: 6.5010 clay

\#python:

$\mathrm{f}=\operatorname{open}(\mathrm{r}$ "Picks.txt","r+")

$\mathrm{p}=$ open $(\mathrm{r}$ "Pos.txt","r+")

ff = open(r"rand4_x_axis.txt","r+")

$\mathrm{g}=$ open(r"rand4_y_axis.txt","r+")

$\mathrm{c}=$ f.readlines ()

$\mathrm{d}=$ p.readlines ()

e=ff.readlines ()

$\mathrm{h}=\mathrm{g} \cdot \operatorname{readlines}()$

picks $=[]$

$\operatorname{pos}=[]$

$\operatorname{val} \_x=[]$

val_y $=[]$ 
for eachLine in c:

\# setup a temporay variable picks.append(float(eachLine))

\# Print the picks list

print (picks)

for eachLine2 in $\mathrm{d}$ :

\# setup a temporay variable pos.append(float(eachLine2))

\# Print the picks list

for eachLine3 in e:

\# setup a temporay variable val_x.append(float(eachLine3))

\# Print the picks list

for eachLine4 in h:

\# setup a temporay variable val_y.append(float(eachLine4))

\# Print the picks list

print (pos)

first_pos=pos[0]

last_pos=len(pos)-1

from gprMax.input_cmd_funcs import *

domain((pos[last_pos])-first_pos, 1.50, 0.002) 
waveform('ricker', 1, 1.0e9, 'my_ricker')

hertzian_dipole('z', 0.02, 1.40, 0, 'my_ricker')

$\operatorname{rx}(0.06,1.40,0)$

$\operatorname{src\_ steps}(0.05,0,0)$

rx_steps $(0.05,0,0)$

box $(0,0,0,($ pos$[$ last_pos])-first_pos, 1.40, 0.002, 'salt')

import numpy as np

VAL_x=np.loadtxt('RAN4_X_axis.txt')

VAL_y=np.loadtxt('RAN4_Y_axis.txt')

for $\mathrm{j}$ in range(40): 'clay')

$\operatorname{box}\left(V A L \_x[j]-0.5, V A L \_y[j]-0.1,0, V A L \_x[j]-0.5+0.50, V A L \_y[j]-0.1+0.02,0.002\right.$,

import math

len $1=1.0$

$\operatorname{len} 2=0.70$

$\operatorname{deg}=[-3.8829,-3.6371,1.7865,-0.0482,-3.1029,-0.0499,-3.5239,-$

4.4503,3.5071,0.6056,4.2961,1.9667,0.8279,3.1540,3.7901,4.8891,-

$4.9948,3.6544,1.1257,3.8995,2.6271,3.4486,-2.9059,0.5229,1.2988,-4.6801,1.1471,-1.3759,-$

$4.5047,-3.0749]$

for $\mathrm{j}$ in range(30):

height $1=(\operatorname{len} 1 / 2) *($ math.sin $($ math.radians $(\operatorname{deg}[j])))$

height2 $=($ len2/2) $*($ math.sin $($ math.radians $(\operatorname{deg}[j])))$

triangle(VAL_x[j], VAL_y[j], 0, VAL_x[j] + (len1/2),VAL_y[j] + height1, 0,VAL_x[j] + len1, VAL_y[j], 0, 0.002, 'clay')

triangle(VAL_x[j]+0.15, VAL_y[j], 0,VAL_x[j] + (len1/2),VAL_y[j] + height2, 0 , VAL_x[j] + len1-0.15, VAL_y[j], 0, 0.002, 'salt')

for $\mathrm{k}$ in range(1000):

box $\left(v a l \_x[k], v a l \_y[k], 0, v a l \_x[k]+0.02\right.$, val_y[k]+0.01, 0.002, 'clay')

for ii in range(1, len(picks)): 
box((pos[ii-1])-first_pos, 0, 0, (pos[ii])-first_pos, 1.40-picks[ii], 0.002, 'clay')

f.close

p.close

\#end_python:

\section{MATLAB script for surface generation}

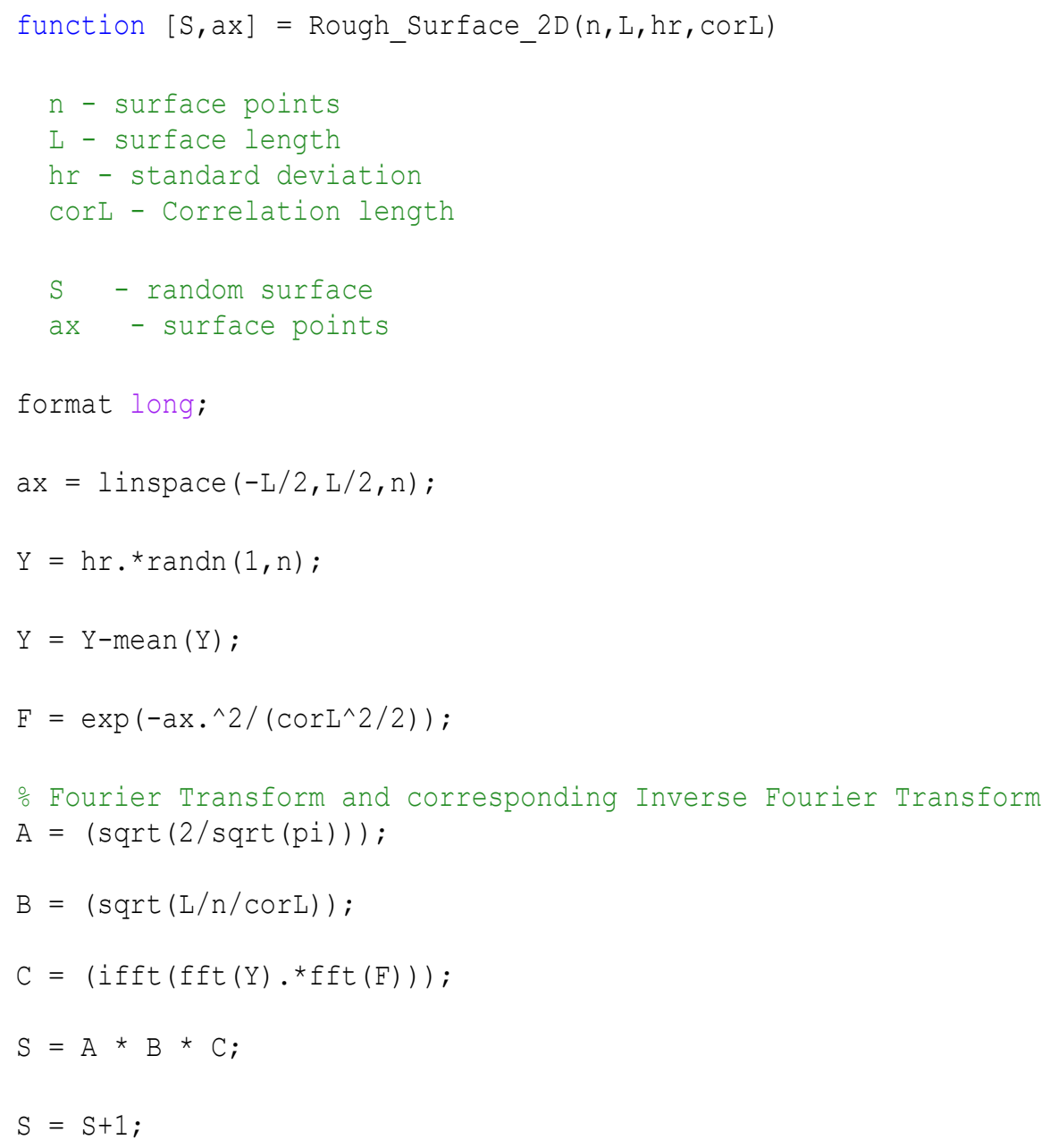




\section{MATLAB script for Gain function}

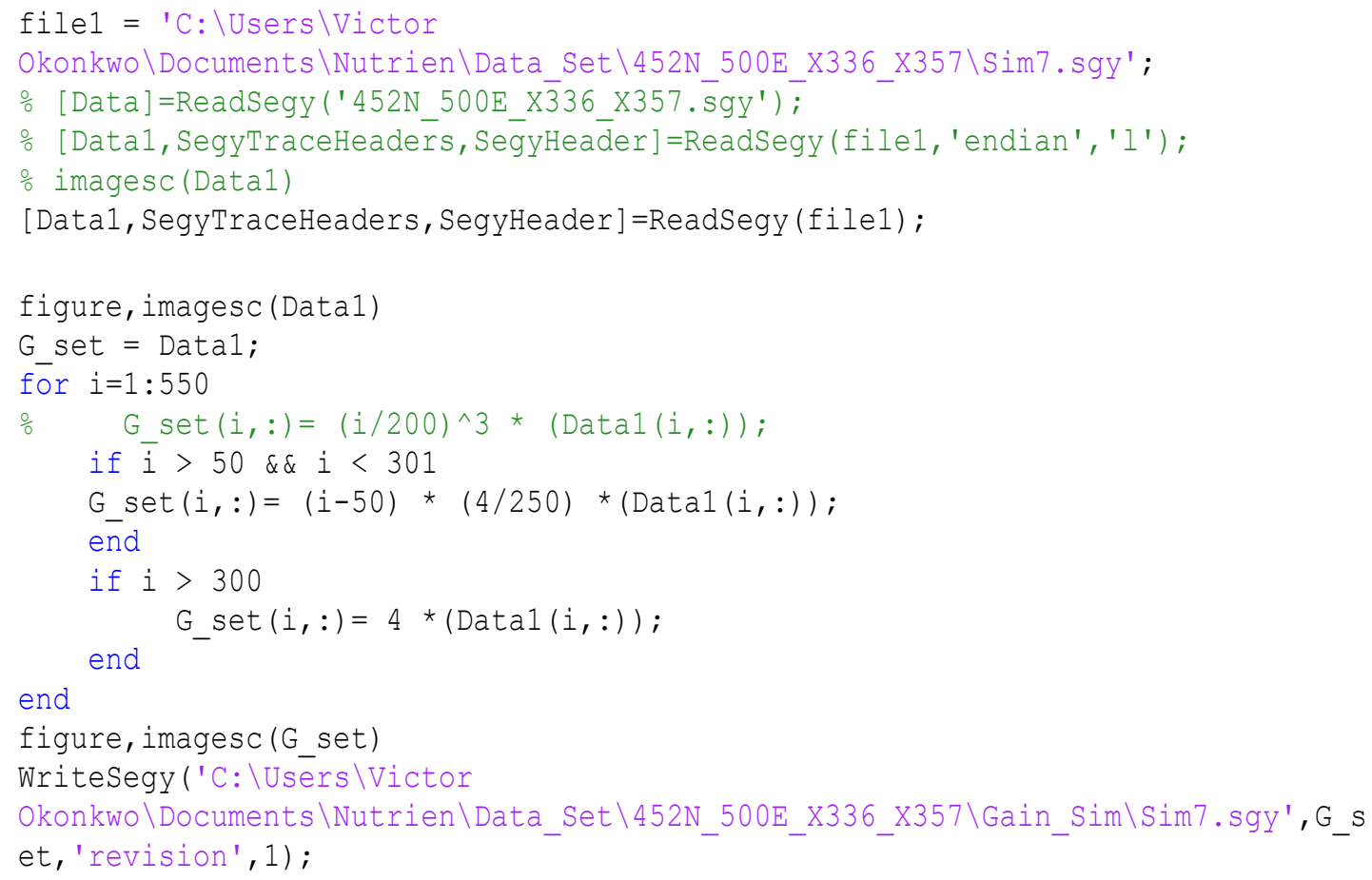

